\title{
THE VARIATION OF THE DE RHAM ZETA FUNCTION
}

\author{
STEVEN ROSENBERG
}

\begin{abstract}
Special values of the zeta function $\zeta(s)$ for the Laplacian on forms $\Delta$ on a compact Riemannian manifold are known to have geometric significance. We compute the variation of these special values with respect to the variation of the metric and write down the Euler-Lagrange equation for conformal variations. The invariant metric on a locally symmetric space is shown to be critical for every local Lagrangian. We also compute the variation of $s^{\prime}(0)$, or equivalently of $\operatorname{det} \Delta$. Finally, flat manifolds are characterized by flatness at a point and a condition on the amplitudes of the eigenforms of $\Delta$.
\end{abstract}

Introduction. In the past ten years, the study of the zeta function of an elliptic operator on a compact Riemannian manifold has led to deep global results, such as a proof of the Atiyah-Singer Index Theorem and the equality of Reidemeister torsion and analytic torsion. In particular, when the operator is exterior differentiation on the de Rham complex of bundle valued forms, then (up to some technicalities) the alternating sum of $\zeta^{q}(0)$, the zeta function on $q$-forms, gives the Euler characteristic, and another linear combination of $\left(\varsigma^{q}\right)^{\prime}(0)$ determines the Reidemeister torsion.

Nevertheless, for fixed $q$ much remains to be known about $\zeta^{q}(0)$ and $\left(\zeta^{q}\right)^{\prime}(0)$. For example, although the linear combinations of $\varsigma^{q}(0)$ and $\left(\varsigma^{q}\right)^{\prime}(0)$ mentioned above are topological invariants, it has not been known how the individual terms depend on the metric. The approach of this paper is to compute the variational equations for $\zeta^{q}(0)$ and $\left(\varsigma^{q}\right)^{\prime}(0)$ as functions on the space of metrics, and to then search for critical metrics.

In more detail, in $\S 1$ we first compute the variation of $\zeta^{q}(0)$ in the direction of an arbitrary symmetric two tensor (Theorem 1.13). This extends results of Ray and Singer [RS]. The Euler-Lagrange equation for conformal variations of the metric is then written down in terms of the heat kernel asymptotics for the Laplacians on forms (Proposition 1.15). We find that on an $m$ dimensional manifold, a metric is critical for conformal variations if and only if a certain $(m-1)$-form is closed (Proposition 1.28). The Euler-Lagrange equations for arbitrary variations can be also calculated by the same techniques. Although directly solving even the conformal Euler-Lagrange equation seems very difficult, we can spot several classes of metrics (e.g., flat metrics, any metric on a surface) that satisfy the full EulerLagrange equations (Lemma 1.17). Using a theorem of Bleecker [B1], we also prove that the invariant metric on a compact locally symmetric space is critical for every natural Lagrangian given by the integral of a local expression (Theorem 1.18). The $\varsigma^{q}(0)$ are always given by such an expression, while $\left(\varsigma^{q}\right)^{\prime}(0)$ is not. The findings of this section indicate that $\zeta^{q}(0)$ is rarely an invariant of any special class of metrics.

Received by the editors October 22, 1985 and, in revised form, January 14, 1986.

1980 Mathematics Subject Classification (1985 Revision). Primary 53C20; Secondary 53C35.

Partially supported by the National Science Foundation. 
The best result we know, due to Terng $[\mathbf{T}]$, is that $\varsigma^{q}(0)$ is an invariant of connected sets of Einstein metrics on a four dimensional manifold. Mostow's Rigidity Theorem precludes using Theorem 1.18 to produce a nontrivial family of metrics with $\varsigma^{q}(0)$ as an invariant.

In $\S 2$ we begin by computing the variational formula for $\left(\varsigma^{q}\right)^{\prime}(0)$ (Theorem 2.5). The formula contains a nonlocal piece together with local terms, the variations of $\varsigma^{q}(s)$ for certain integral values of $s$.

A similar formula for $\left(\varsigma^{q}\right)^{\prime}(0)$ itself is in Cheeger's work $[\mathbf{C}]$. These local terms regularize a formal expression, known to the physicists, for the variation of $\left(\varsigma^{q}\right)^{\prime}(0)$. This formal expression is in fact valid in dimension two, and we show that the only critical metric for conformal variations of $\left(\zeta^{q}\right)^{\prime}(0)$ is the one of constant curvature (Theorem 2.7). Above dimension two the Euler-Lagrange equation necessarily involves the Green's function for the various Laplacians, so no local equation for critical metrics can be found (Proposition 2.13).

We also investigate the interplay between formal and rigorous expressions for a localized zeta function $\zeta(s, x)$ for $x$ on the manifold $M$. For example, if $\left\{\phi_{n}\right\}$ is an orthonormal basis of eigenfunctions of the Laplacian, then for all $x, \sum\left|\phi_{n}(x)\right|^{2}$, the formal expression for $\zeta(0, x)$ diverges. However, if $\operatorname{dim} M$ is odd,

$$
\sum\left(\left|\phi_{n}(x)\right|^{2}-\left|\phi_{n}(y)\right|^{2}\right)=0 \quad \text { for } x, y \in M,
$$

whenever the sum exists (Theorem 2.11). This can be thought of as a generalization of properties of the trigonometric polynomials. Moreover, flat manifolds can be characterized by (i) $\sum\left(\left|\phi_{n}(x)\right|^{2}-\left|\phi_{n}(y)\right|^{2}\right)$ exists on 0 -, 1-, and 2-forms and (ii) flatness at one point.

The author began this research as a NATO Postdoctoral Fellow. During the writing and revising of this paper, he received helpful suggestions from Professors M. F. Atiyah, J. Cheeger, D. Fried, R. Palais, D. Quillen, and C. L. Terng.

1. Let $M$ be an oriented compact Riemannian manifold. In this section we will study certain special values of zeta functions associated to the metric on $M$. In particular, we will see how the special values vary as the metric is deformed. Since the relevant values depend on whether $M$ is even or odd dimensional, we will assume that $M$ is even dimensional and just indicate the necessary changes for odd dimensional manifolds.

To begin, we will review the basic properties of the zeta function and geometric significance of the special values. The metric $\rho$ determines an inner product on forms via the Hodge star operator $*$, and with respect to this inner product the exterior derivative $d$ has an adjoint $\delta= \pm * d *$. The Laplacian on $q$-forms is given by $\Delta^{q}=\Delta_{\rho}^{q}=d \delta+\delta d$. Let $\left\{\lambda_{n}\right\}$ be the spectrum of $\Delta^{q}$, repeated with multiplicity. The zeta function for $q$-forms is defined to be

$$
\zeta^{q}(s)=\zeta_{\rho}^{q}(s)=\sum_{\lambda_{n} \neq 0} \frac{1}{\lambda_{n}^{s}}
$$

for $s \in$ C. Note that $\varsigma^{q}(s)=\varsigma^{m-q}(s)$ on an $m$ dimensional manifold, since $* \Delta=$ $\Delta *$.

The convergence of the zeta function and its analytic continuation are controlled by the heat operator $e^{-t \Delta^{q}}$ on $q$-forms. This operator is trace class on the space 
of $L^{2} q$-forms with trace $\operatorname{Tr}\left(e^{-t \Delta^{q}}\right)=\sum_{n} e^{-\lambda_{n} t}$. The definition of the gamma function then gives

$$
\zeta^{q}(s)=\frac{1}{\Gamma(s)} \int_{0}^{\infty} t^{s-1} \operatorname{Tr}\left(e^{-t \Delta^{q}}-P^{q}\right) d t,
$$

where $P^{q}$ is the projection of the $L^{2} q$-forms onto the harmonic $q$-forms. Since $\operatorname{Tr}\left(e^{-t \Delta^{q}}\right)$ is $O\left(t^{-m / 2}\right)$ as $t \downarrow 0$ (see 1.2), the Mellin transform (1.1) shows that $\varsigma^{q}(s)$ is analytic for $\operatorname{Re}(s)>m / 2$. Conversely, starting with the zeta function, one can recover first the trace of the heat operator (except for the harmonic forms contribution) by an inverse Mellin transform. Fourier inversion then gives the nonzero spectrum with multiplicity. Thus the zeta function encodes all the spectral information of $\Delta^{q}$ except for the $q$ th Betti number.

For the analytic continuation of $\varsigma^{q}(s)$, we first recall that the heat operator has a smooth kernel $e^{q}(t, x, y)$ with an asymptotic expansion

$$
e^{q}(t, x, y)=\frac{e^{-r^{2}(x, y) / 4 t}}{(4 \pi t)^{m / 2}}\left(\sum_{k=0}^{N} C_{k}^{q}(x, y) t^{k}+O\left(t^{N}\right)\right) \quad \text { as } t \downarrow 0 .
$$

Here $r(x, y)$ is the geodesic distance between nearby points $x$ and $y, m$ is the dimension of $M, N$ is an integer greater than $m / 2$, and $C_{k}^{q}(x, y)$ is a $(q, q)$-double form on $M$. For later purposes, we remark that the coefficients $C_{k}^{q}$ are defined inductively to be the unique solution of

$$
\begin{gathered}
\Delta C_{k-1}^{q}(x, y)+\nabla_{r(d / d r)} C_{k}^{q}(x, y)+\left(k+\frac{r}{4 g} \frac{d g}{d r}\right) C_{k}^{q}(x, y)=0, \\
C_{-1}^{q}(x, y) \equiv 0
\end{gathered}
$$

where $g$ is the determinant of the metric at $y$ and all differentiations are in the $x$ variable $[\mathbf{P}]$. The significance of the asymptotic expansion is that $\operatorname{tr} C_{k}^{q}(x, x)$ is explicitly computable from the curvature tensor and its covariant derivatives at $x$. (Recall that the trace of a $(q, q)$-double form $\alpha \otimes \beta(x, y)$ at $x$ is $\alpha(x) \wedge * \beta(x)$.) Writing the right side of (1.1) as $\int_{0}^{1}+\int_{0}^{\infty}$ and plugging

$$
\begin{aligned}
\operatorname{Tr}\left(e^{-t \Delta^{q}}\right) & =\int_{M} \operatorname{Tr} e^{q}(t, x, x) \\
& =\frac{1}{(4 \pi t)^{m / 2}}\left(\sum_{k=0}^{N} t^{k} \int_{M} \operatorname{tr} C_{k}^{q}(x, x)+O\left(t^{N}\right)\right), \quad t \in(0,1),
\end{aligned}
$$

into the first integral, we get

$$
\begin{aligned}
\varsigma^{q}(s)= & \frac{1}{(4 \pi)^{m / 2} \Gamma(s)}\left(\sum_{k=0}^{N} \frac{1}{s+k-m / 2} \int_{M} \operatorname{tr} C_{k}^{q}(x, x)\right) \\
& -\frac{\beta_{q}}{s \Gamma(s)}+\frac{1}{\Gamma(s)} \cdot R(s),
\end{aligned}
$$

where $R(s)$ is analytic for $\operatorname{Re}(s)>-N-1$ and $\beta_{q}$ is the $q$ th Betti number. (For more details, see $[\mathbf{R S}]$.) Since $\Gamma(s)$ has poles at the nonpositive integers with residue 
$(-1)^{n}(n !)^{-1}$ at $-n$,

$$
\begin{aligned}
\varsigma^{q}(0) & =\frac{1}{(4 \pi)^{m / 2}} \int_{M} \operatorname{tr} C_{m / 2}^{q}(x, x)-\beta_{q}, \\
\varsigma^{q}(-k) & =\frac{(-1)^{k} k !}{(4 \pi)^{m / 2}} \int_{M} \operatorname{tr} C_{k+m / 2}^{q}(x, x), \quad k \in \mathbf{Z}^{+}, \\
\operatorname{res} \varsigma^{q}(k) & =\frac{1}{(4 \pi)^{m / 2}(k-1) !} \int_{M} \operatorname{tr} C_{k-m / 2}^{q}(x, x), \quad k \in \mathbf{Z}, 0<k \leq \frac{m}{2} .
\end{aligned}
$$

For odd dimensional manifolds, the relevant values of the zeta function would be $\left\{k+\frac{1}{2}: k \in \mathbf{Z}\right.$ and $\left.k<\frac{1}{2} \operatorname{dim} M\right\}$. Notice that $\zeta^{q}(0)=-\beta_{q}$ in odd dimensions, so in this case the zeta function encodes all of the spectrum of $\Delta^{q}$.

While the geometric expressions for $\int_{M} \operatorname{tr} C_{k}^{q}$ become overwhelmingly complicated as $k$ increases (see e.g. $[\mathbf{G 1}]$ ), the heat equation approach for the index of the de Rham complex [ABP] easily gives

$$
\begin{aligned}
& \sum_{q}(-1)^{q} \varsigma^{q}(k)=0, \quad k \in \mathbf{Z}^{-}, \\
& \sum_{q}(-1)^{q} \operatorname{res} \varsigma^{q}(k)=0, \quad 0<k \leq m / 2, k \in \mathbf{Z} .
\end{aligned}
$$

For $k=0$, it is a remarkable result of Patodi ( $[\mathbf{P}]$; see also $[\mathbf{M S}]$ for $m=2)$ that the Euler form $E$ is given pointwise by

$$
E(x)=\frac{1}{(4 \pi)^{m / 2}} \sum(-1)^{q} \operatorname{tr} C_{m / 2}^{q}(x, x) .
$$

By the Gauss-Bonnet Theorem,

$$
\sum_{q}(-1)^{q} \varsigma^{q}(0)=\int_{M} E(x)-\chi(m)=0 .
$$

It appears to be difficult to understand how the individual terms $\operatorname{tr} C_{m / 2}^{q}(x, x)$ contribute to the Euler form. What we can do is compute how the contribution of the global term $\int_{M} \operatorname{tr} C_{m / 2}^{q}$ to the Euler characteristic depends on the metric. The local question will be discussed later (see (1.19)-(1.21)).

To calculate the variation of $\int_{M} \operatorname{tr} C_{m / 2}^{q}$ as a function of the metric, it is equivalent by (1.5) to compute the variation of $\varsigma^{q}(0)$. The set of all metrics on $M$ forms an open cone within the vector space of symmetric two tensors, $S^{2} T^{*} M$, so the tangent space to the metric $\rho$ is $S^{2} T^{*} M$. Thus the variation $\delta \zeta_{\rho}^{q}(0)$ in the direction $\rho_{1} \in S^{2} T^{*} M$ is given by $d /\left.d u\right|_{u=0 \zeta_{\rho+u \rho_{1}}^{q}(0) \text {. (We hope the two uses of the symbol }}$ $\delta$ will cause no confusion.) Since the zeta function depends smoothly upon the metric at the regular values, we may write

$$
\begin{aligned}
\delta \zeta_{\rho}^{q}(0) & =\left.\left(\left.\frac{d}{d u}\right|_{u=0} \zeta_{\rho+u \rho_{1}}^{q}(s)\right)\right|_{s=0} \\
& =\left.\left(\left.\frac{1}{\Gamma(s)} \int_{0}^{\infty} t^{s-1} \frac{d}{d u}\right|_{u=0} \operatorname{Tr}\left(e^{-t \Delta_{u}^{q}}-P_{u}^{q}\right) d t\right)\right|_{s=0}
\end{aligned}
$$

where $\Delta_{u}=\Delta_{\rho+u \rho_{1}}$ and $P_{u}^{q}=P_{\rho+u \rho_{1}}^{q}$. By the Hodge Theorem, $(d / d u) \operatorname{Tr}\left(P_{u}^{q}\right)=0$. Moreover, Ray and Singer show that

$$
\frac{d}{d u} \operatorname{Tr}\left(e^{-t \Delta_{u}}\right)=-t \operatorname{Tr}\left(\dot{\Delta}_{u} e^{-t \Delta_{u}}\right)
$$


for $\dot{\Delta}_{u}=d \Delta_{u} / d u$. If we set $\dot{*}=d *_{u} / d u$ and $\alpha=*^{-1} \dot{*}$, then it is easy to check that $\dot{\Delta}=\delta \alpha d-d \alpha \delta-\alpha \delta d+d \delta \alpha$. Since $\operatorname{Tr}(A B)=\operatorname{Tr}(B A)$ for $A$ trace class and $B$ bounded,

$$
\begin{aligned}
\operatorname{Tr}\left(\delta \alpha d e^{-t \Delta^{q}}\right) & =\operatorname{Tr}\left(e^{-t \Delta^{q} / 2} \delta \alpha d e^{-t \Delta^{q} / 2}\right) \\
& =\operatorname{Tr}\left(\alpha d e^{-t \Delta^{q}} \delta\right)=\operatorname{Tr}\left(\alpha d \delta e^{-t \Delta^{q+1}}\right) .
\end{aligned}
$$

Using similar manipulations for the other terms in $\operatorname{Tr}\left(\dot{\Delta} e^{-t \Delta}\right)$, we get

$$
\begin{aligned}
\delta \zeta_{\rho}^{q}(0)=\frac{1}{\Gamma(s)} \int_{0}^{\infty} t^{s} \operatorname{Tr}\left(-\alpha d \delta e^{-t \Delta^{q+1}}\right. & +\alpha \delta d e^{-t \Delta^{q}} \\
& \left.+\alpha \delta d e^{-t \Delta^{q-1}}-\alpha d \delta e^{-t \Delta^{q}}\right) d t \quad \text { at } s=0 .
\end{aligned}
$$

In (1.9) we have set $u=0$ and dropped $u$ as a subscript.

We claim that we can replace the integral in (1.9) by the same integral just taken from zero to one. First of all, $\alpha d \delta e^{-t \Delta^{q+1}}$ has kernel $\alpha d \delta e^{q+1}(t, x, y)$, where $\alpha d \delta$ acts on the $x$ variable. If $\left\{\phi_{n}\right\}$ is an orthonormal basis of $L^{2} L^{2}(q+1)$-forms consisting of eigenforms of $\Delta^{q+1}$ with eigenvalues $\left\{\lambda_{n}\right\}$, then

$$
e^{q+1}(t, x, y)=\sum_{n} e^{-\lambda_{n} t} \phi_{n}(x) \otimes \phi_{n}(y)
$$

in the $C^{\infty}$ topology on $M \times M$. By the Hodge Decomposition Theorem on $(q+1)$ forms, we may assume that each $\phi_{n}$ is either harmonic, an eigenform of $d \delta$, or an eigenform of $\delta d$. Since $\alpha$ acts fiberwise in the bundle of $(q+1)$-forms, it is easy to check that $\operatorname{Tr}\left(\alpha d \delta e^{-t \Delta^{q+1}}\right)=O\left(\sum_{n} e^{-\lambda_{n}^{\prime} t}\right)=O\left(e^{-\lambda t}\right)$, for $\left\{\lambda_{n}^{\prime}\right\}$ the eigenvalues of $d \delta$ and $\lambda$ the first nonzero eigenvalue of $\Delta^{q+1}$. Thus the integral from one to infinity in (1.9) is analytic for all $s \in \mathbf{C}$. Since $1 / \Gamma(s)$ is zero at $s=0$, this establishes the claim.

Thus we arrive at the formula, essentially due to Ray and Singer,

$$
\begin{aligned}
\delta \zeta^{p}(0)=\frac{1}{\Gamma(s)} \int_{0}^{1} t^{s}\left[\int _ { M } \operatorname { t r } \left(-\alpha d \delta e^{q+1}(t, x, y)+\alpha \delta d e^{q}(t, x, y)\right.\right. & \\
& \left.\left.+\alpha \delta d e^{q-1}(t, x, y)-\alpha d \delta e^{q}(t, x, y)\right)\right] d t \quad \text { at } s=0 .
\end{aligned}
$$

The integral over $M$ can be simplified by the following lemma.

LEMMA 1.11. Let $\omega$ be a p-form in the variable $x$ on a Riemannian manifold of dimension $m$. Let $r$ be the geodesic distance function and let all differentiations be in the $x$ variable. Then

$$
\begin{aligned}
& \left.d \delta\left(e^{-r^{2}(x, y) / 4 t} \omega\right)\right|_{y=x}=\frac{p}{2 t} \omega+d \delta \omega, \\
& \left.\delta d\left(e^{-r^{2}(x, y) / 4 t} \omega\right)\right|_{y=x}=\frac{m-p}{2 t} \omega+\delta d \omega .
\end{aligned}
$$

PROOF. Choose a normal coordinate system $\left(x^{1}, \ldots, x^{m}\right)$ centered at $y$ with metric matrix $\left(g_{i j}\right)$. Let $\nabla_{i}=\nabla_{\partial / \partial x^{i}}$ be the covariant derivative in the $x^{i}$ direction 
and let $\nabla^{i}=g^{i k} \nabla_{k}$ (with summation convention). For $f(x)=e^{-r^{2}(x, y) / 4 t}$, we have

$$
\left.\nabla_{k} f\right|_{x=y}=\frac{\partial f}{\partial x^{k}}=\left.f(x)\left(-\frac{1}{4 t}\right) \frac{\partial}{\partial x^{k}} r^{2}(x, y)\right|_{y=x}
$$

It is well known that in normal coordinates $r^{2}(x, y)=g_{i k} x^{i} x^{k}$, so $\left.\nabla_{k} f\right|_{x=y}=0$. Using $g_{i k}=\delta_{i k}$ at $x=y$, we similarly see that $\left.\nabla^{i} \nabla_{k} f\right|_{x=y}=0$ if $i \neq k$ and $-\left.\nabla^{k} \nabla_{k} f\right|_{x=y}=1 / 2 t$ (no summation convention).

For $\omega=\omega_{k_{1} \cdots k_{p}} d x^{k_{1}} \wedge \cdots \wedge d x^{k_{p}}$, we have

$$
(d \omega)_{k_{1} \cdots k_{p+1}}=\sum_{\nu=1}^{p+1}(-1)^{\nu-1} \nabla_{k_{\nu}} \omega_{k_{1} \cdots \hat{k}_{\nu} \cdots k_{p+1}}
$$

$\left(\hat{k}_{\nu}\right.$ means $k_{\nu}$ is omitted $)$ and

$$
(\delta \omega)_{k_{1} \cdots k_{p-1}}=-\nabla^{i} \omega_{i k_{1} \cdots k_{p-1}}
$$

[de $\mathbf{R}, \S 26]$. Thus at $x=y$,

$$
\begin{aligned}
((d \delta) f \omega)_{k_{1} \cdots k_{p}}= & \sum_{\nu=1}^{p}(-1)^{\nu} \nabla_{k_{\nu}} \nabla^{i}(f \omega)_{i k_{1} \ldots \hat{k}_{\nu} \cdots k_{p}} \\
= & \sum_{\nu=1}^{p}(-1)^{\nu}\left(\nabla_{k_{\nu}} \nabla^{i} f\right) \omega_{i k_{1} \cdots \hat{k}_{\nu} \cdots k_{p}} \\
& +\sum_{\nu=1}^{p}(-1)^{\nu} f \nabla_{k_{\nu}} \nabla^{i} \omega_{i k_{1} \cdots \hat{k}_{\nu} \cdots k_{p}} \\
= & \sum_{\nu=1}^{p}(-1)^{\nu}\left(\nabla_{k_{\nu}} \nabla^{k_{\nu}} f\right) \omega_{k_{\nu} k_{1} \cdots \hat{k}_{\nu} \cdots k_{p}}+f d \delta \omega \\
= & -\sum_{\nu=1}^{p}\left(\nabla_{k_{\nu}} \nabla^{k_{\nu}} f\right) \omega_{k_{1} \cdots k_{p}}+d \delta \omega \\
= & \frac{p}{2 t} \omega+d \delta \omega .
\end{aligned}
$$

Also at $x=y$,

$$
\begin{aligned}
((\delta d) f \omega)_{k_{1} \cdots k_{p}}= & -\nabla^{i} \nabla_{i}(f \omega)_{k_{1} \cdots k_{p}}-\sum_{\nu=1}^{p}(-1)^{\nu} \nabla^{i} \nabla_{k_{\nu}}(f \omega)_{i k_{1} \cdots \hat{k}_{\nu} \cdots k_{p}} \\
= & \left(-\nabla^{i} \nabla_{i} f\right) \omega_{k_{1} \cdots k_{p}}+f\left(-\nabla^{i} \nabla_{i} \omega\right)_{k_{1} \cdots k_{p}} \\
& -\sum_{\nu=1}^{p}(-1)^{\nu}\left(\nabla^{i} \nabla_{k_{\nu}} f\right) \omega_{i k_{1} \cdots \hat{k}_{\nu} \cdots k_{p}} \\
& -f \sum_{\nu=1}^{p}(-1)^{\nu} \nabla^{i} \nabla_{k_{\nu}} \omega_{i k_{1} \cdots \hat{k}_{\nu} \cdots k_{p}} \\
= & \frac{m}{2 t} \omega+\delta d \omega-\frac{p}{2 t} \omega .
\end{aligned}
$$

We now substitute the asymptotic expansion in for each heat kernel in (1.10) and apply the lemma. If we abbreviate $\int_{M} \operatorname{tr} \alpha d \delta C_{k}^{q}(x, x)$ by $\int_{M} \alpha d \delta C_{k}^{q}$, etc., the 
result is

$$
\begin{aligned}
& \delta \zeta_{\rho}^{q}(0)=\frac{1}{(4 \pi)^{m / 2} \Gamma(s)} \int_{0}^{1} t^{s}\left[\int_{M}-\alpha \sum_{k=0}^{N} d \delta C_{k}^{q+1} t^{k-m / 2}\right. \\
&-\int_{M} \alpha\left(\frac{q+1}{2 t}\right) \sum_{k=0}^{N} C_{k}^{q+1} t^{k-m / 2} \\
&+\int_{M} \alpha \sum_{k=0}^{N} \delta d C_{k}^{q} t^{k-m / 2} \\
&+\int_{M} \alpha\left(\frac{m-q}{2 t}\right) \sum_{k=0}^{N} C_{k}^{q} t^{k-m / 2} \\
&+\int_{M} \alpha \sum_{k=0}^{N} \delta d C_{k}^{q-1} t^{k-m / 2} \\
&+\int_{M} \alpha\left(\frac{m-q+1}{2 t}\right) \sum_{k=0}^{N} C_{k}^{q-1} t^{k-m / 2} \\
&-\int_{M} \alpha \sum_{k=0}^{N} d \delta C_{k}^{q} t^{k-m / 2} \\
&\left.-\int_{M} \alpha\left(\frac{q}{2 t}\right) \sum_{k=0}^{N} C_{k}^{q} t^{k-m / 2}+O\left(t^{N-m / 2}\right)\right] d t
\end{aligned}
$$$$
\text { at } s=0 \text {. }
$$

The error term comes from applying $d \delta$ and $\delta d$ to the error term in (1.2). It follows from the estimates used in constructing the $C_{k}^{q}$ in $[\mathbf{P}]$ that the new error is indeed $O\left(t^{N-m / 2}\right)$. We can now perform the $t$ integration. This gives, with some terms omitted,

$$
\begin{aligned}
& \delta \zeta_{\rho}^{q}(0)=\frac{1}{(4 \pi)^{m / 2} \Gamma(s)}\left[\sum_{k=0}^{N} \frac{1}{s-k-m / 2+1}\left(\int_{M}-\alpha d \delta C_{k}^{q+1}\right)\right. \\
&\left.-\sum_{k=0}^{N}\left(\frac{q+1}{2}\right) \frac{1}{s+k-m / 2}\left(\int_{M} \alpha C_{k}^{q+1}\right)+\cdots+O(1)\right] \\
& \text { at } s=0 .
\end{aligned}
$$

Note we choose $N$ and $s$ so that $s+k-m / 2$ and $s+k-m / 2-1$ do not equal -1 . $\Gamma(s)$ has a simple pole at $s=0$ with residue 1 , so nonzero terms occur on the right in (1.12) only when $k=m / 2-1$ is in the first sum, $k=m / 2$ in the second sum, etc. This gives the final variational formula. 
THEOREM 1.13. Let $M$ be an oriented compact manifold of even dimension $m$ with metric $\rho$. Then

$$
\begin{aligned}
& \delta \zeta_{\rho}^{q}(0)=\frac{1}{(4 \pi)^{m / 2}}\left[-\int_{M} \alpha d \delta C_{m / 2-1}^{q+1}-\frac{q+1}{2} \int_{M} \alpha C_{m / 2}^{q+1}\right. \\
& +\int_{M} \alpha \delta d C_{m / 2-1}^{q}+\left(\frac{m}{2}-q\right) \int_{M} \alpha C_{m / 2}^{q}+\int_{M} \alpha \delta d C_{m / 2-1}^{q-1} \\
& \left.+\frac{m-q+1}{2} \int_{M} \alpha C_{m / 2}^{q-1}-\int_{M} \alpha d \delta C_{m / 2-1}^{q}\right] \text {. }
\end{aligned}
$$

Here $\alpha d \delta C_{m / 2-1}^{q+1}$ means $\operatorname{tr} \alpha d \delta C_{m / 2-1}^{q+1}$ with $\alpha d \delta$ applied in the $x$ variable, etc.

The only dependence of $\delta \zeta_{\rho}^{q}(0)$ in the direction of variation is in $\alpha$, i.e., in the variation of $*_{\rho}$. This is a bit surprising since $\Delta_{p}= \pm d *_{\rho} d *_{\rho} \pm *_{\rho} d *_{\rho} d$ depends on the metric both in $*_{\rho}$ and in manifold derivatives of $*_{\rho}$. It is also surprising that information from dimensions $q \pm 1$ appears (see Proposition 1.28).

If we vary $\rho$ in its own direction by setting $\rho_{1}=\lambda^{2} \rho$ for $\lambda \in \mathbf{R}-\{0\}$, then

$$
\dot{*}=\left.\frac{d}{d u}\right|_{u=0}\left(1+u \lambda^{2}\right)^{m / 2-q_{*}}
$$

on $q$-forms, and so $\alpha=(m / 2-q) \lambda^{2}$. Id on $q$-forms. Also, under the change $\rho \rightarrow$ $\left(1+u \lambda^{2}\right) \rho=\rho_{u}$, the eigenvalues $\left\{\lambda_{n}\right\}$ of $\Delta^{q}$ go to the eigenvalues $\left\{\left(1+u \lambda^{2}\right)^{-2} \lambda_{n}\right\}$ of $\Delta_{\rho_{u}}$. Thus $\zeta_{\rho_{u}}^{q}(s)=\left(1+u \lambda^{2}\right)^{2 s} \zeta_{\rho}^{q}(s)$, so $\delta \zeta_{\rho}^{q}(0)=0$ for this variation. This direction therefore leads to global relations $A_{q}$ among the $C_{k}^{q}$.

COROLlary 1.14 .

$$
\begin{aligned}
\left(A_{q}\right) \quad 0= & \left(\frac{m}{2}-q-1\right)\left[-\int_{M} d \delta C_{m / 2-1}^{q+1}-\frac{q+1}{2} \int_{M} C_{m / 2}^{q+1}\right] \\
& +\left(\frac{m}{2}-q\right)\left[\int_{M} \delta d C_{m / 2-1}^{q}+\left(\frac{m}{2}-q\right) \int_{M} C_{m / 2}^{q}-\int_{M} d \delta C_{m / 2-1}^{q}\right] \\
& +\left(\frac{m}{2}-q+1\right)\left[\int_{M} \delta d C_{m / 2-1}^{q-1}+\frac{m-q+1}{2} \int_{M} C_{m / 2}^{q-1}\right], \\
\left(B_{q}\right) \quad 0= & \left(\frac{m}{2}-q+1\right)\left[\int_{M} \delta d C_{m / 2-1}^{q-1}+\frac{m-q+1}{2} \int_{M} C_{m / 2}^{q-1}\right] \\
& +\left(\frac{m}{2}-q\right)\left[-\int_{M} d \delta C_{m / 2-1}^{q}-\frac{q}{2} \int_{M} C_{m / 2}^{q}\right] .
\end{aligned}
$$

$B_{0}$ is the same as $A_{0} . B_{q}$ then follows by subtracting $B_{q-1}$ from $A_{q}$. In other words, $A_{q}=B_{q-1}+B_{q}$, so the $B_{q}$ would seem to be a more basic identity.

It is not the case that $A_{q}$ is the integral of a pointwise identity. In fact, a metric $\rho$ is a critical point for $\zeta^{q}(0)$, considered as a function on the set of all metrics conformal to $\rho$, if and only if $A_{q}$ vanishes pointwise. To see this, we let $\rho_{i}=\delta_{i} \rho$, where $\delta_{i}$ approaches a delta function at a fixed $x \in M$ as $i$ goes to infinity. The corresponding $\alpha_{i}$ is $(m / 2-q) \cdot \delta_{i} \cdot \operatorname{Id}$, so letting $i$ go to infinity gives the result. 
Proposition 1.15. A metric $\rho$ is a critical point for $\zeta^{q}(0)$ within its conformal class if and only if for all $x \in M$,

$$
\begin{aligned}
A_{q}(x) \quad 0= & \left(\frac{m}{2}-q-1\right)\left(-d \delta C_{m / 2-1}^{q+1}-\left(\frac{q+1}{2}\right) C_{m / 2}^{q+1}\right)(x) \\
& +\left(\frac{m}{2}-q\right)\left(\delta d C_{m / 2-1}^{q}+\left(\frac{m}{2}-q\right) C_{m / 2}^{q}-d \delta C_{m / 2-1}^{q}\right)(x) \\
& +\left(\frac{m}{2}-q+1\right)\left(\delta d C_{m / 2-1}^{q-1}+\left(\frac{m-q+1}{2}\right) C_{m / 2}^{q-1}\right)(x) .
\end{aligned}
$$

Here $d \delta C_{m / 2-1}^{q+1}(x)$ means $\left.\operatorname{tr} d \delta C_{m / 2-1}^{q+1}(x, y)\right|_{y=x}$, with $d \delta$ applied in the $x$ variable.

We now show the existence of a metric $g$ on the 4-torus $T$, conformally equivalent to the flat metric, for which $A_{q}(x) \not \equiv 0$ for some $q$. If no such metric exists, then $\varsigma^{q}(0)$ is an invariant for the conformal class of the flat metric. Let $R_{i j k l}$ be the Riemann curvature tensor for $g$, and let

$$
R^{2}=R_{i j k l} R_{i j k l} ; \quad \rho_{j l}=R_{i j i l} ; \quad \rho^{2}=\rho_{i j} \rho_{i j} ; \quad \tau=\rho_{i i}
$$

with summation convention. Then using (1.5)

$$
\begin{gathered}
0=32 \pi^{2} \chi(T)=\int_{T} R^{2}-4 \rho^{2}+\tau^{2} d v o l \\
16 \pi^{2} \zeta_{g}^{0}(0)+1=\int_{T} R^{2}-2 \rho^{2}+5 \tau^{2} d v o l \\
16 \pi^{2} \zeta_{q}^{1}(0)+4=\int_{T}-22 R^{2}+172 \rho^{2}-40 \tau^{2} d v o l .
\end{gathered}
$$

The first equation is Avez's formula, and the other equations are in [G2]. The conformal invariance of $\zeta^{q}(0)$ would imply that the second and third equations are also zero, since the integrands vanish for the flat metric. This implies $\int R^{2}=\int \rho^{2}=$ $\int \tau^{2}=0$, so $R_{i j k l} \equiv 0$. In other words, $g$ must be flat, which is a contradiction.

The Euler-Lagrange equations for arbitrary variations can also be derived from Theorem 1.13. One computes $\alpha$ for an infinitesimal variation in each of the possible $m(m+1) / 2$ directions at a point $x \in M$, say in normal coordinates at $x$. Of course, this only yields the equations in coordinate dependent form, and it may be difficult to interpret the equations invariantly. For our purposes, it is enough to realize that the Euler-Lagrange equations in normal coordinates will be expressions in the $C_{k}^{q}$ occuring in $A_{q}$ and their derivatives.

Some classes of metrics which are critical points for $\zeta^{q}(0)$ can be written down immediately.

LEMMA 1.17. The following metrics are critical points for arbitrary variations of $\varsigma^{q}(0)$ :

(a) flat metrics,

(b) any metric on a two dimensional manifold,

(c) the standard $G$-invariant metric on a homogeneous space $G / H, G$ compact and $H$ closed, whose isotropy representation is irreducible,

(d) Einstein metrics on a four dimensional manifold.

Proof. (a) A flat metric has the same heat kernel asymptotics as Euclidean space. In Euclidean space, $C_{0}^{q}(x, y)=\sum_{I, J} d x^{I} \otimes d y^{J}$ in multi-index notation, and $C_{k}^{q}(x, y) \equiv 0$ for $k>0$. 
(b) In two dimensions,

$$
\varsigma^{0}(0)=\varsigma^{2}(0)=\frac{1}{4 \pi} \int_{M} \frac{\tau}{6} d v o l-1
$$

[MS]. The Gauss-Bonnet Theorem shows that $\varsigma^{0}(0)$ and $\varsigma^{2}(0)$ are independent of the metric. $\zeta^{1}(0)$ is then independent of the metric by (1.6). For just conformal variations we could also prove (b) by noting that $A_{0}(x)$ is the same as (1.3) at $x=y$.

(c) The $G$-invariant metric on an isotropy irreducible homogeneous space is critical for every variational problem which is invariant under the natural action of the diffeomorphism group and which is scale invariant (i.e. independent of $\rho \mapsto \lambda^{2} \rho$ ). This is a theorem of Bleecker [B1].

(d) In four dimensions, $\varsigma^{q}(0)=\int_{M} A R^{2}+B \rho^{2}+C \tau^{2} d v o l$, where $A, B, C$ are constants depending only on $q[\mathbf{G} \mathbf{2}]$.

It is a result of Terng $[\mathbf{T}]$ that Einstein metrics satisfy $\delta \int_{M} R^{2}=\delta \int_{M} \rho^{2}=$ $\delta \int_{M} \tau^{2}=0$.

Thus $\varsigma^{q}(0)$ is an invariant of the connected components of Einstein metrics on a four dimensional manifold. In particular, $\varsigma^{q}(0)$ is an invariant on the family of metrics produced by Yau's solution of the Calabi conjecture on a $K 3$ surface. No other nontrivial families of Einstein metrics seem to be known. In any case, the Einstein metrics are known to form only a finite dimensional space [BE].

We can obtain a new class of critical metrics by using Bleecker's result along with a Hirzebruch Proportionality argument from [CGW]. First we need some definitions. We call a Lagrangian $\mathcal{L}(g)$ local if it is given by integration over $M$ of a top dimensional form $f(x) \operatorname{dvol}(x)$, where $f(x)$ is a smooth function of the metric and its derivatives at $x$. We also say that a Lagrangian $\mathcal{L}(g)$ has weight $k$ if scaling the metric $g \mapsto \lambda^{2} g$ has the effect $\mathcal{L}(g) \mapsto \lambda^{-k} \mathcal{L}(g)$. As usual, if $\mathcal{L}(g)$ is not scale invariant (i.e. of weight 0 ), then we restrict our attention to metrics of volume one.

THEOREM 1.18. Let $\mathcal{L}(g)$ be a local Lagrangian of fixed weight $k$ on the space of metrics. Let $M=\Gamma \backslash G / K$ be the quotient of a symmetric space $G / K$ by a discrete, torsion free, cocompact subgroup $\Gamma$. Then the canonical $G$-invariant metric on $M$ of volume one is a critical point for $\mathcal{L}(g)$.

PROOF. Since $\mathcal{L}(g)$ is local, the standard calculus of variations techniques will yield a symmetric matrix of Euler-Lagrange equations of the form $\left(P_{i j}(x, g)\right) \equiv 0$, where $P(x, g)$ is a local expression in the metric at $x \in M$. (This is under the assumption that $\mathcal{L}(g)$ is scale invariant. If not, the constraint on the volume of $g$ leads to $\left(P_{i j}(x, g)\right)=C \cdot\left(g_{i j}(x)\right)$ for some constant $C$.) If $G / \bar{K}$ is compact, the $\Gamma$ invariance of the metric implies that $G / K$ has the same Euler-Lagrange equations as $\Gamma \backslash G / K$. By Bleecker's theorem, the canonical metric on $G / K$ is critical, so the equations will be satisfied on $G / K$ and hence on $\Gamma \backslash G / K$.

Assume $G / K$ is noncompact. Since $f(x) \operatorname{dvol}(x)$ is a well defined form, the EulerLagrange equations will be invariant expressions in the metric. By $[\mathbf{A B P}, \S 2]$, in normal coordinates $f(x)$ is an expression in the curvature tensor and its covariant derivatives. These equations are independent of $x$, since $G$ acts transitively on $G / K$ by isometries, and in fact the covariant derivative terms vanish since the curvature tensor is parallel. 
The volume form is of weight $-m=-\operatorname{dim} M$, so each $P_{i j}(x, g)$ is of weight $k+m$. The components $R_{i j k l}$ of the curvature tensor are of weight 2 , so $P_{i j}(x, g)$ must be a sum of terms, each of which is the product of $(k+m) / 2$ curvature components. (In particular, $k+m$ must be even and $k \geq-m$.)

On the compact dual symmetric space $G^{\prime} / K$, the curvatures in the invariant metric $g^{\prime}$ are minus those of $G / K$, so $P_{i j}\left(x, g^{\prime}\right)$ on $G^{\prime} / K$ is $(-1)^{(k+m) / 2} P_{i j}(x, g)$ for the invariant metric $g$ on $\Gamma \backslash G / K$. In the unconstrained case, $\left(P_{i j}\left(x, g^{\prime}\right)\right) \equiv 0$ on $G^{\prime} / K$ by Bleecker's theorem, and hence $\left(P_{i j}(x, g)\right) \equiv 0$ on $\Gamma \backslash G / K$. In the constrained case, we have $\left(P_{i j}\left(e K, g^{\prime}\right)\right)=C \cdot\left(\delta_{i j}\right)$ in normal coordinates at the identity coset $e K \in G^{\prime} / K$, and hence $\left(P_{i j}(e K, g)\right)=C \cdot\left(\delta_{i j}\right)$ in normal coordinates at $e K$ in $\Gamma \backslash G / K$.

Thus the canonical metric on $\Gamma \backslash G / K$ is critical for $\int_{M} C_{k}^{q}(g)$ for every $k$ and therefore critical for $\varsigma^{q}(k)$ (or its residue) for $k=m / 2, m / 2-1, \ldots$. In particular, the integrands $A_{q}(x)$ and $B_{q}(x)$ in Corollary 1.14 vanish pointwise for this metric.

We would now like to examine $A_{q}(x)$ more closely. Since $A_{q}(x)$ is a top dimensional form whose integral is zero, $A_{q}(x)$ is exact by de Rham's Theorem. In fact, finding a form $\theta$ with $d \theta=A_{q}$ will lead us to a simpler Euler-Lagrange equation for conformal variations (Proposition 1.28). To find $\theta$, we first have to define a pointwise zeta function and compute its variation.

DEFinition 1.19. Let $\omega$ be a differential form on a manifold $M$. For $x \in M$, define $\omega^{2}(x)$ by $\omega(x) \wedge * \omega(x)=\omega^{2}(x) \operatorname{dvol}(x)$.

Now let $\left\{\phi_{n}\right\}$ be an orthonormal basis of $L^{2} q$-forms on $M$ consisting of eigenforms of the Laplacian. We define the pointwise zeta function by

$$
\varsigma^{q}(s, x)=\sum_{\lambda_{n} \neq 0} \frac{\phi_{n}^{2}(x)}{\lambda_{n}^{s}}, \quad x \in M
$$

We quickly sketch the convergence of $\varsigma^{q}(s, x)$. Let $\left\{\omega_{i}\right\}_{i=1}^{k}$ denote an orthonormal basis of harmonic $q$-forms. We define $\beta_{q}(x)$, the $q$ th local Betti number at $x$, to be $\sum_{i=1}^{k} \omega_{i}^{2}(x)$. Note that $\int_{M} \beta_{q}(x) d \operatorname{vol}(x)=\beta_{q}$, the $q$ th Betti number. Then

$$
\varsigma^{q}(s, x)=\frac{1}{\Gamma(s)} \int_{0}^{\infty} t^{s-1}\left(* \operatorname{tr}\left(e^{q}(t, x, x)\right)-\beta_{q}(x)\right) d t
$$

(for us, taking the trace yields a top dimensional form, so we need the extra star operator). This gives the range of convergence of $\zeta^{q}(s, x)$ and its analytic continuation as with $\varsigma^{q}(s)$. Thus $\varsigma^{q}(0, x)=* \operatorname{tr} C_{k}^{q}(x, x)-\beta_{q}(x)$, and $\varsigma^{q}(s)=\int_{M} \varsigma^{q}(x, s) d v o l(x)$.

The calculation leading up to Theorem 1.13 had two global steps, equations (1.7) and (1.8). In computing

$$
\delta \varsigma^{q}(0, x)=\frac{1}{\Gamma(s)} \int_{0}^{1} t^{s-1}\left(\left.\frac{d}{d u}\right|_{u=0} * \operatorname{tr} e_{u}^{q}(t, x, x)\right) d t \quad \text { at } s=0
$$


(1.7) must be replaced by the more complicated term [RS, pp. 198-199]

$$
\begin{aligned}
\left.\frac{d}{d u}\right|_{u=0} \operatorname{tr} e_{u}^{q}(t, x, x) \\
\quad=\int_{0}^{t} \operatorname{tr}\left[\left((\delta \alpha d-\alpha \delta d-d \alpha \delta+\alpha d \delta) e^{\left.\left.-t^{\prime} \Delta^{q}\right)_{x} e^{q}\left(t-t^{\prime}, x, y\right)\right] d t^{\prime}}\right.\right. \\
=\int_{0}^{t} \operatorname{tr}\left[(\delta \alpha d-\alpha \delta d-d \alpha \delta+\alpha d \delta)_{x} e^{q}(t, x, y)\right] d t^{\prime} \\
=t \operatorname{tr}\left[(\delta \alpha d-\alpha \delta d-d \alpha \delta+\alpha d \delta)_{x} e^{q}(t, x, y)\right]
\end{aligned}
$$

by the semigroup property of the heat kernel. The subscript $x$ denotes the variable of differentiation. Since we are working locally now, we cannot move $\alpha$ out in front as in (1.8). Thus the variation of the pointwise zeta function involves manifold derivatives of $\alpha$. We could obtain a formula for $\delta \zeta(0, x)$ by proving a lemma analogous to (1.11) for $d \alpha \delta$ and $\delta \alpha d$, but for our purposes we will just consider variations in the directions of the metric, i.e. $\alpha=(m / 2-q) \lambda^{2} \cdot$ Id on $q$-forms. In this case, (1.20) reduces to $-\lambda^{2} t \operatorname{tr}\left(\Delta_{x}^{q} e^{q}(t, x, y)\right)$. Therefore for these variations we get

$$
0=\delta\left(\varsigma^{q}(0, x) d \operatorname{vol}(x)\right)=\frac{1}{\Gamma(s)} \int_{0}^{1}-t^{s} \operatorname{tr}\left(\Delta_{x}^{q} e^{q}(t, x, y)\right) d t
$$

Using the product rule for the Laplacian (just add the equations in 1.11), we obtain as before

$$
0=\frac{m}{2} \operatorname{tr} C_{m / 2}^{q}(x, x)+\operatorname{tr} \Delta_{x} C_{m / 2-1}^{q}(x, y)
$$

This local identity is in itself nothing new, as it is just (1.3) at $x=y$. The real interest is in (1.21). For $\alpha=(m / 2-q) \lambda^{2} \cdot \mathrm{Id}$, we claim that

$$
\begin{aligned}
-\operatorname{tr} \Delta_{x}^{q} e^{q}(t, x, y)+\operatorname{tr}(- & \alpha d \delta e^{q+1}(t, x, y)+\alpha \delta d e^{q}(t, x, y) \\
& \left.+\alpha \delta d e^{q-1}(t, x, y)-\alpha d \delta e^{q}(t, x, y)\right)=d \theta
\end{aligned}
$$

for an explicitly computable $(m-1)$ dimensional form $\theta=\theta(t)$. We will also produce an asymptotic expansion $\theta \sim\left(1 /(4 \pi t)^{m / 2}\right) \sum_{k} \theta_{k}(x) t^{k}$. Assuming this, we will have

$$
\begin{aligned}
0=\frac{1}{\Gamma(s)} \int_{0}^{1} t^{s}\left(d \theta(t)-\operatorname{tr}\left(-\alpha d \delta e^{q+1}(t,\right.\right. & x, y)+\alpha \delta d e^{q}(t, x, y) \\
& \left.\left.+\alpha \delta d e^{q-1}(t, x, y)-\alpha d \delta e^{q}(t, x, y)\right)\right) d t
\end{aligned}
$$

Substituting the asymptotic expansion for $\theta$ and proceding as in Theorem 1.13 will give us

$$
0=d \theta_{m / 2}(x)-A_{q}(x) .
$$

To write down $\theta$, we first recall the Hodge Decomposition for smooth $q$-forms, $C^{\infty} \Lambda^{q}=H^{q} \oplus d C^{\infty} \Lambda^{q-1} \oplus \delta C^{\infty} \Lambda^{q+1}$, where $H^{q}$ denotes the harmonic $q$-forms. We choose an orthonormal basis of eigenforms of $\Delta^{q}$ of the form $\left\{\tilde{u}_{n}\right\} \cup\left\{u_{n}^{\prime}\right\} \cup$ $\left\{u_{n}^{\prime \prime}\right\}$, where $\left\{\tilde{u}_{n}\right\},\left\{u_{n}^{\prime}\right\}$, and $\left\{u_{n}^{\prime \prime}\right\}$ are bases of $H^{q}, d C^{\infty} \Lambda^{q-1}$, and $\delta C^{\infty} \Lambda^{q+1}$ respectively. We denote the corresponding eigenvalues by $0,\left\{\lambda_{n}^{\prime}\right\}$, and $\left\{\lambda_{n}^{\prime \prime}\right\}$. We let $\left\{\tilde{w}_{n}\right\},\left\{w_{n}^{\prime}\right\}$, and $\left\{w_{n}^{\prime \prime}\right\}$ denote the corresponding eigenforms for $(q-1)$-forms, with eigenvalues $0,\left\{\nu_{n}^{\prime}\right\}$, and $\left\{\nu_{n}^{\prime \prime}\right\}$, and for $(q+1)$-forms we use the eigenforms $\left\{\tilde{z}_{n}\right\},\left\{z_{n}^{\prime}\right\}$, and $\left\{z_{n}^{\prime \prime}\right\}$, with eigenvalues $0,\left\{\psi_{n}^{\prime}\right\}$, and $\left\{\psi_{n}^{\prime \prime}\right\}$. In what follows, we will denote $\alpha(x) \wedge * \beta(x)$ by $\operatorname{tr} \alpha \otimes \beta(x, y)$ even if $\alpha$ and $\beta$ have different degrees. 
LEMMA 1.23. For $\alpha=(m / 2-q) \cdot$ Id on $q$-forms,

$$
\begin{aligned}
-\operatorname{tr} \Delta_{x}^{q} e^{q}(t, x, y)+\operatorname{tr}( & -(\alpha d \delta)_{x} e^{q+1}(t, x, y)+(\alpha \delta d)_{x} e^{q}(t, x, y) \\
+ & \left.(\alpha \delta d)_{x} e^{q-1}(t, x, y)-(\alpha d \delta)_{x} e^{q}(t, x, y)\right)=d \theta
\end{aligned}
$$

where

$$
\theta=-(m / 2-q-1) \operatorname{tr} d_{y} e^{q}(t, x, y)-(m / 2-q+1) \operatorname{tr} d_{y} e^{q-1}(t, x, y) .
$$

PROOF. Let $\left\{\lambda_{n}\right\}$ denote all the eigenvalues of $\Delta^{q}$. The difference of the traces in $(1.23)$ is

$$
\begin{aligned}
& -\sum e^{-\lambda_{n} t} \lambda_{n} u_{n} \wedge * u_{n}-\left(\frac{m}{2}-q-1\right) \sum e^{-\psi_{n}^{\prime} t} \psi_{n}^{\prime} z_{n} \wedge * z_{n} \\
& +\left(\frac{m}{2}-q\right) \sum e^{-\lambda_{n}^{\prime \prime} t} \lambda_{n}^{\prime \prime} w_{n}^{\prime \prime} \wedge * w_{n}^{\prime \prime}+\left(\frac{m}{2}-q+1\right) \sum e^{-\nu_{n}^{\prime \prime} t} \nu_{n}^{\prime \prime} w_{n}^{\prime \prime} \wedge * w_{n}^{\prime \prime} \\
& \quad-\left(\frac{m}{2}-q\right) \sum e^{-\lambda_{n}^{\prime} t} \lambda_{n}^{\prime} u_{n}^{\prime} \wedge * u_{n}^{\prime}
\end{aligned}
$$

since $\Delta \mid \delta C^{\infty} \Lambda^{*}=\delta d$ and $\Delta \mid d C^{\infty} \Lambda^{*}=d \delta$. Splitting the first term into a sum over $\left\{\lambda_{n}^{\prime}\right\}$ and $\left\{\lambda_{n}^{\prime \prime}\right\}$ gives

$$
\begin{aligned}
& \left(\frac{m}{2}-q-1\right)\left[\sum e^{-\lambda_{n}^{\prime \prime} t} \lambda_{n}^{\prime \prime} u_{n}^{\prime \prime} \wedge * u_{n}^{\prime \prime}-\sum e^{-\psi_{n}^{\prime} t} \psi_{n}^{\prime} z_{n}^{\prime} \wedge * z_{n}^{\prime}\right] \\
& +\left(\frac{m}{2}-q+1\right)\left[\sum e^{-\nu_{n}^{\prime \prime} t} \nu_{n}^{\prime \prime} w_{n}^{\prime \prime} \wedge * w_{n}^{\prime \prime}-\sum e^{-\lambda_{n}^{\prime} t} \lambda_{n}^{\prime} u_{n}^{\prime} \wedge * u_{n}^{\prime}\right] .
\end{aligned}
$$

It is well known that $d+\delta$ taking even forms to odd forms is an isomorphism for each nonzero eigenspace of the Laplacian on even forms. The Hodge Decomposition then implies that the set $\left\{\lambda_{n}^{\prime \prime}\right\}$, with each $\lambda_{n}^{\prime \prime}$ repeated with multiplicity, is equal to $\left\{\psi_{n}^{\prime}\right\}$, repeated to multiplicity. By the $d+\delta$ isomorphism, $d u_{n}^{\prime \prime}$ is an eigenform for $d \delta$ with eigenvalue $\lambda_{n}^{\prime \prime}$. Moreover, the $L^{2}$ norm of $d u_{n}^{\prime \prime}$ is

$$
\left\|d u_{n}^{\prime \prime}\right\|^{2}=\int d u_{n}^{\prime \prime} \wedge * d u_{n}^{\prime \prime}=\int \delta d u_{n}^{\prime \prime} \wedge * u_{n}^{\prime \prime}=\lambda_{n}^{\prime \prime}
$$

Similarly $d u_{i}^{\prime \prime}$ is orthogonal to $d u_{j}^{\prime \prime}$ if $i \neq j$. We can therefore replace each $z_{n}^{\prime}$ by $\left(\lambda_{n}^{\prime \prime}\right)^{-1 / 2} d u_{n}^{\prime \prime}$ as an orthogonal basis of $d C^{\infty} \Lambda^{q}$. By the same argument for $\left\{w_{n}^{\prime \prime}\right\}$ and $\left\{u_{n}^{\prime}\right\}$, (1.24) becomes

$$
\begin{aligned}
& \left(\frac{m}{2}-q-1\right)\left[\sum e^{-\lambda_{n}^{\prime \prime} t} \lambda_{n}^{\prime \prime} u_{n}^{\prime \prime} \wedge * u_{n}^{\prime \prime}-\sum e^{-\lambda_{n}^{\prime \prime} t} d u_{n}^{\prime \prime} \wedge * d u_{n}^{\prime \prime}\right] \\
& +\left(\frac{m}{2}-q+1\right)\left[\sum e^{-\nu_{n}^{\prime \prime} t} \nu_{n}^{\prime \prime} w_{n}^{\prime \prime} \wedge * w_{n}^{\prime \prime}-\sum e^{-\nu_{n}^{\prime \prime} t} d w_{n}^{\prime \prime} \wedge * d w_{n}^{\prime \prime}\right] .
\end{aligned}
$$

Now

$$
\theta=-(m / 2-q-1) \sum e^{-\lambda_{n}^{\prime \prime} t} u_{n}^{\prime \prime} \wedge * d u_{n}^{\prime \prime}-\left(\frac{m}{2}-q+1\right) \sum e^{-\nu_{n}^{\prime \prime} t} w_{n}^{\prime \prime} \wedge * d w_{n}^{\prime \prime},
$$

since $d_{y}$ kills the $H^{*}$ and $d C^{\infty} \Lambda^{*}$ summands in $e^{*}(t, x, y)$. We compute

$$
\begin{aligned}
d \theta= & \left(\frac{m}{2}-q-1\right)\left[-\sum e^{-\lambda_{n}^{\prime \prime} t} d u_{n}^{\prime \prime} \wedge * d u_{n}^{\prime \prime}-(-1)^{q} \sum e^{-\lambda_{n}^{\prime \prime} t} u_{n}^{\prime \prime} \wedge d * d u_{n}^{\prime \prime}\right] \\
& +\left(\frac{m}{2}-q+1\right)\left[-\sum e^{-\nu_{n}^{\prime \prime} t} d w_{n}^{\prime \prime} \wedge * d w_{n}^{\prime \prime}-(-1)^{q-1} \sum e^{-\nu_{n}^{\prime \prime} t} w_{n}^{\prime \prime} \wedge d * d w_{n}^{\prime \prime}\right] \\
= & \left(\frac{m}{2}-q-1\right)\left[-\sum e^{-\lambda_{n}^{\prime \prime} t} d u_{n}^{\prime \prime} \wedge * d u_{n}^{\prime \prime}+\sum e^{-\lambda_{n}^{\prime \prime} t} u_{n}^{\prime \prime} \wedge * \delta d u_{n}^{\prime \prime}\right] \\
& +\left(\frac{m}{2}-q+1\right)\left[-\sum e^{-\nu_{n}^{\prime \prime} t} d w_{n}^{\prime \prime} \wedge * d w_{n}^{\prime \prime}+\sum e^{-\nu_{n}^{\prime \prime} t} w_{n}^{\prime \prime} \wedge * \delta d w_{n}^{\prime \prime}\right] .
\end{aligned}
$$


By the definition of $\left\{u_{n}^{\prime \prime}\right\}$ and $\left\{w_{n}^{\prime \prime}\right\},(1.25)$ and (1.26) agree.

Thus

$$
\begin{aligned}
\theta= & -\left(\frac{m}{2}-q-1\right) \operatorname{tr} d_{y}\left(\frac{e^{-r^{2}(x, y) / 4 t}}{(4 \pi t)^{m / 2}}\left(\sum_{k=0}^{N} C_{k}^{q}(x, y) t^{k}+O\left(t^{N}\right)\right)\right) \\
& -\left(\frac{m}{2}-q+1\right) \operatorname{tr} d_{y}\left(\frac{e^{-r^{2}(x, y) / 4 t}}{(4 \pi t)^{m / 2}}\left(\sum_{k=0}^{N} C_{k}^{q-1}(x, y) t^{k}+O\left(t^{N}\right)\right)\right) .
\end{aligned}
$$

Since $\left.d_{y} r^{2}(x, y)\right|_{x=y}=0$ and $d O\left(t^{N}\right)=O\left(t^{N}\right)$ as before, we have

$$
\begin{aligned}
\theta(x, t)= & -\left(\frac{m}{2}-q-1\right) \frac{1}{(4 \pi t)^{m / 2}} \sum_{k=0}^{N} \operatorname{tr} d_{y} C_{k}^{q} \cdot t^{k} \\
& -\left(\frac{m}{2}-q+1\right) \frac{1}{(4 \pi t)^{m / 2}} \sum_{k=0}^{N} \operatorname{tr} d_{y} C_{k-1}^{q} t^{k}+O\left(t^{N}\right) .
\end{aligned}
$$

By (1.22), this gives us the desired expression for $A_{q}(x)$ :

$$
(4 \pi)^{m / 2} A_{q}(x)=d\left[-\left(\frac{m}{2}-q-1\right) \operatorname{tr} d_{y} C_{m / 2}^{q}-\left(\frac{m}{2}-q+1\right) \operatorname{tr} d_{y} C_{m / 2}^{q-1}\right] .
$$

This equation leads to a much simpler formulation for the Euler-Lagrange equation for a metric critical for $\varsigma^{q}(0)$ within its conformal class. Note that as expected the equation in (1.28) only depends on information in dimension $q$.

PROPOSITION 1.28. A metric is critical for $\varsigma^{q}(0)$ within its conformal class if and only if

$$
(m / 2-q-1) \operatorname{tr} d_{y} C_{m / 2}^{q}+(m / 2-q+1) \operatorname{tr} d_{y} C_{m / 2}^{q-1}
$$

is closed. Equivalently, a metric is critical if and only if

$$
\begin{aligned}
& (m / 2-q-1)\left(\operatorname{tr} d_{x} d_{y} C_{m / 2}^{q}-\operatorname{tr}(\delta d)_{x} C_{m / 2}^{q}\right) \\
& \quad+(m / 2-q+1)\left(\operatorname{tr}(d \delta)_{x} C_{m / 2}^{q}-\operatorname{tr} \delta_{x} \delta_{y} C_{m / 2}^{q}\right) \equiv 0 .
\end{aligned}
$$

ProOF. We first claim we can replace $d_{y} C_{m / 2}^{q-1}$ in (1.27) by $\delta_{x} C_{m / 2}^{q}$. The identity $\delta \Delta^{q}=\Delta^{q-1} \delta$ yields $\delta e^{-t \Delta^{q}}=e^{-t \Delta^{q-1}} \delta$. Then for any smooth $q$-form $\phi$,

$$
\begin{aligned}
\delta e^{-t \Delta^{q}} \phi(x) & =\int \delta_{x} e^{q}(t, x, y) \wedge *_{y} \phi(y), \\
e^{-t \Delta^{q-1}} \delta \phi(x) & =\int e^{q-1}(t, x, y) \wedge *_{y} \delta_{y} \phi(y)=\int d_{y} e^{q-1}(t, x, y) \wedge *_{y} \phi(y) .
\end{aligned}
$$

Thus $\delta_{x} e^{q}(t, x, y)=d_{y} e^{q-1}(t, x, y)$. Plugging in the asymptotics for the heat kernels gives the claim.

Next, we note that if $\alpha$ and $\beta$ are $q$-forms,

$$
d(\alpha \wedge * \beta)=d \alpha \wedge * \beta+\alpha \wedge * * d * \beta=\operatorname{tr} d_{x}(\alpha \otimes \beta)-\operatorname{tr} \delta_{y}(\alpha \otimes \beta)
$$

(this uses the even dimensionality of the manifold). $C_{m / 2}^{q}$ is approximated arbitrarily closely in the $C^{\infty}$ topology by linear combinations of double forms $\alpha(x) \otimes \beta(y)$, 
so (1.27) becomes

$$
\begin{aligned}
(4 \pi)^{m / 2} A_{q}(x)= & -(m / 2-q-1)\left[\operatorname{tr} d_{x} d_{y} C_{m / 2}^{q}-\operatorname{tr} \delta_{y} d_{y} C_{m / 2}^{q}\right] \\
& -(m / 2-q+1)\left[\operatorname{tr} d_{x} \delta_{x} C_{m / 2}^{q}-\operatorname{tr} \delta_{y} \delta_{x} C_{m / 2}^{q}\right]
\end{aligned}
$$

Finally, as above we can replace $(\delta d)_{y} C_{m / 2}^{q}$ by $(\delta d)_{x} C_{m / 2}^{q}$. Since a metric is critical within its conformal class if and only if $A_{q}(x)=0$, this finishes the proof.

We end this section by remarking that all the results for $\varsigma^{q}(0)$ can be extended to $\varsigma^{q}(-n), n \in \mathbf{Z}^{+}$, and $\operatorname{res} \varsigma^{q}(n), 0<n \leq m / 2$. One simply picks out the appropriate $C_{k}$ in (1.12) at $s= \pm n$. More specifically, let $A_{n}^{q}$ denote the integrals in brackets in Theorem 1.13, with each subscript $m / 2$ replaced by $m / 2+n$. Then

$$
\begin{aligned}
\delta_{\zeta}(-n) & =\frac{(-1)^{n} n !}{(4 \pi)^{m / 2}} \cdot A_{n}^{q}, \quad n \in \mathbf{Z}^{+}, \\
\delta \operatorname{res} \zeta(n) & =\frac{1}{(4 \pi)^{m / 2}(n-1) !} \cdot A_{-n}^{q}, \quad 0<n \leq m / 2, n \in \mathbf{Z} .
\end{aligned}
$$

It is also easy to verify that if $m=\operatorname{dim} M$ is odd, then

$$
\delta \operatorname{res} \varsigma^{q}\left(\frac{m}{2}-n\right)=\frac{1}{(4 \pi)^{m / 2} \Gamma(m / 2-n)} A_{n-m / 2}^{q}, \quad n \in \mathbf{Z}^{+} \cup\{0\} .
$$

For future use, we note that (1.29) and (1.30) are equivalent to

$$
\delta \operatorname{res} \Gamma\left(\frac{m}{2}-n\right) \varsigma^{q}\left(\frac{m}{2}-n\right)=\frac{1}{(4 \pi)^{m / 2}} A_{n-m / 2}^{q}
$$

2. In this section we will consider the variation of $\left(\varsigma^{q}\right)^{\prime}(0)=d \varsigma^{q}(s) /\left.d s\right|_{s=0}$. The motivation is again a topological invariance associated to $\varsigma^{\prime}(0)$. Recall that exterior differentiation and the Hodge star on $M$ extend to operators on forms with values in a bundle $E$ associated to an orthogonal representation of $\pi_{1}(M)$. Therefore we can consider zeta functions associated to a metric on $M$ and such a bundle $E$. If $E$ is acyclic, i.e., $E$ has trivial cohomology groups, then Ray and Singer conjectured that

$$
\frac{1}{2} \sum_{q=1}^{m}(-1)^{q} q\left(\varsigma^{q}\right)^{\prime}(0)=\ln \tau(M, E)
$$

where $\tau(M, E)$ is the Reidemeister torsion. This was proven independently by Cheeger and Müller.

Throughout this section $M$ can be either odd or even dimensional. All the computations will be done for ordinary $q$-forms, but the results extend immediately to forms with values in a bundle, acyclic or not, associated to a representation of $\pi_{1}(M)$.

The calculation of the variation proceeds much as before. We have

$$
\begin{aligned}
\delta\left(\varsigma^{q}\right)^{\prime}(0) & =\left.\left.\frac{d}{d s}\right|_{s=0} \frac{d}{d u}\right|_{u=0} \frac{1}{\Gamma(s)} \int_{0}^{\infty} t^{s-1} \operatorname{Tr}\left(e^{-t \Delta_{u}^{q}}-P_{u}^{q}\right) d t \\
& =\left.\frac{d}{d s}\right|_{s=0} \frac{1}{\Gamma(s)} \int_{0}^{\infty} t^{s} \operatorname{Tr}\left(\dot{\Delta} e^{-t \Delta}\right) d t
\end{aligned}
$$

To keep the notation manageable, we give a definition. 
DEFINITION 2.1. Let

$$
I(\alpha, q)=-\alpha d \delta e^{-t \Delta^{q+1}}+\alpha \delta d e^{-t \Delta^{q}}+\alpha \delta d e^{-t \Delta^{q-1}}-\alpha d \delta e^{-t \Delta^{q}} .
$$

Previously, we broke the integral up into contributions from zero to one and from one to infinity, but here it is helpful to introduce a cutoff parameter $p$. Thus

$$
\begin{aligned}
\delta\left(\varsigma^{q}\right)^{\prime}(0)= & \left.\frac{d}{d s}\right|_{s=0}\left[\frac{1}{\Gamma(s)} \int_{0}^{p} t^{s} \operatorname{Tr} I(\alpha, q) d t+\frac{1}{\Gamma(s)} \int_{p}^{\infty} t^{s} \operatorname{Tr} I(\alpha, q) d t\right] \\
= & \frac{1}{\Gamma(s)} \int_{0}^{p} t^{s} \ln t \operatorname{Tr} I(\alpha, q) d t+\left(\frac{1}{\Gamma(s)}\right)^{\prime} \int_{0}^{p} t^{s} \operatorname{Tr} I(\alpha, q) d t \\
& +\frac{1}{\Gamma(s)} \int_{p}^{\infty} t^{s} \ln t \operatorname{Tr} I(\alpha, q) d t+\left(\frac{1}{\Gamma(s)}\right)^{\prime} \int_{p}^{\infty} t^{s} \operatorname{Tr} I(\alpha, q) d t
\end{aligned}
$$

at $s=0$.

The integral in the third term is analytic at $s=0$, so the zero of $1 / \Gamma(s)$ at $s=0$ kills this term. We now substitute the heat kernel asymptotics into $I(\alpha, q)$ in the first two integrals and apply Lemma 1.11. The result is, for $N \gg 0$,

$$
\begin{aligned}
\delta\left(\varsigma^{q}\right)^{\prime}(0)= & \frac{1}{\Gamma(s)} \sum_{k=0}^{N} \int_{0}^{p} t^{s+k-m / 2} \ln t d t\left(\frac{1}{(4 \pi)^{m / 2}} \int_{M}\left(-\alpha d \delta C_{k}^{q+1}-\cdots\right)\right) \\
& +\frac{1}{\Gamma(s)} \int_{0}^{p} t^{s} \ln t O\left(t^{N-m / 2}\right) d t \\
& +\left(\frac{1}{\Gamma(s)}\right)^{\prime} \sum_{k=0}^{N} \int_{0}^{p} t^{s+k-m / 2} d t\left(\frac{1}{(4 \pi)^{m / 2}} \int_{M}\left(-\alpha d \delta C_{k}^{q+1}-\cdots\right)\right) \\
& +\left(\frac{1}{\Gamma(s)}\right)^{\prime} \int_{0}^{p} t^{s-m / 2} O\left(t^{N}\right) d t \\
& +\left(\frac{1}{\Gamma(s)}\right)^{\prime} \int_{p}^{\infty} t^{s} \operatorname{Tr} I(\alpha, q) d t \quad \text { at } s=0 .
\end{aligned}
$$

We denote the terms on the right in (2.2) by $A, B, C, D$, and $E$ respectively. The integral over $M$ in $A$ and $C$ is

$$
\begin{aligned}
\int- & \alpha d \delta C_{k}^{q+1}-\left(\frac{q+1}{2 t}\right) \alpha C_{k}^{q+1}+\alpha \delta d C_{k}^{q}+\left(\frac{m-q}{2 t}\right) \alpha C_{k}^{q} \\
& +\alpha \delta d C_{k}^{q-1}+\left(\frac{m-q+1}{2}\right) \alpha C_{k}^{q-1}-\alpha d \delta C_{k}^{q}-\left(\frac{q}{2 t}\right) \alpha C_{k}^{q}
\end{aligned}
$$

Since

$$
\int_{0}^{p} t^{\beta} \ln t d t=\frac{p^{\beta+1}}{\beta+1}\left(\ln p-\frac{1}{\beta+1}\right)
$$

for $\operatorname{Re}(\beta)>-1, A$ becomes

$$
\begin{aligned}
\frac{1}{\Gamma(s)} & \sum_{k=0}^{N} \frac{p^{s+k-m / 2}}{s+k-m / 2}\left(\ln p-\frac{1}{s+k-m / 2}\right)\left(\frac{1}{(4 \pi)^{m / 2}} A_{k-m / 2}^{q}\right) \\
& +\frac{1}{\Gamma(s)} \frac{p^{s+N-m / 2+1}}{s+N-m / 2+1}\left(\ln p-\frac{1}{s+N-m / 2+1}\right) \\
& \cdot\left(\frac{1}{(4 \pi)^{m / 2}} \int_{M}\left(-\alpha d \delta C_{N}^{q+1}+\alpha \delta d C_{N}^{q}+\alpha \delta d C_{N}^{q-1}-\alpha d \delta C_{N}^{q}\right)\right) \quad \text { at } s=0 .
\end{aligned}
$$


$A_{n}^{q}$ is defined at the end of $\S 1$. If $M$ is odd dimensional, $k$ never equals $m / 2$, so $A=0$ at $s=0$. Otherwise, the only contribution to (2.2) from $A$ is

$$
\ln p\left(\frac{1}{(4 \pi)^{m / 2}} A_{0}^{q}\right)+\lim _{s \rightarrow 0}-\frac{1}{s}\left(\frac{1}{(4 \pi)^{m / 2}} A_{0}^{q}\right) .
$$

The apparent pole term in (2.3) is cancelled by $C$. Since $d /\left.d s\right|_{s=0} \frac{1}{\Gamma(s)}=1, C$ is

$$
\begin{aligned}
& \sum_{k=0}^{N} \frac{p^{s+k-m / 2}}{s+k-m / 2} \cdot \frac{1}{(4 \pi)^{m / 2}} A_{k-m / 2}^{q}+\frac{p^{s+N-m / 2+1}}{s+N-m / 2+1} \\
& \cdot \frac{1}{(4 \pi)^{m / 2}} \int_{M}\left(-\alpha d \delta C_{N}^{q+1}+\alpha \delta d C_{N}^{q}+\alpha \delta d C_{N}^{q-1}-\alpha d \delta C_{N}^{q}\right) \quad \text { at } s=0 .
\end{aligned}
$$

Notice the pole term at $k=m / 2$.

The integral in $B$ is

$$
O\left(\frac{p^{N+s+1-m / 2}}{N+s+1-m / 2}\left(\ln p-\frac{1}{N+s+1-m / 2}\right)\right)
$$

for fixed $p$, so $B$ vanishes at $s=0$. Clearly $D$ contributes an $O\left(p^{N+1-m / 2}\right)$ term. Finally, there is no problem setting $s=0$ in $E$. In the following theorem, we have used (1.31) to replace $A_{k-m / 2}^{q}$ terms by $\delta \operatorname{res} \Gamma(m / 2-k) \varsigma^{q}(m / 2-k)$.

THEOREM 2.5. The variation of $\left(\varsigma^{q}\right)^{\prime}(0)$ is given by

$$
\begin{aligned}
\delta\left(\varsigma^{q}\right)^{\prime}(0)= & (\ln p) \delta \varsigma^{q}(0)+\sum_{\substack{k=0 \\
k \neq m / 2}}^{N} \frac{p^{k-m / 2}}{k-m / 2} \delta \operatorname{res} \Gamma\left(\frac{m}{2}-k\right) \varsigma^{q}\left(\frac{m}{2}-k\right) \\
& +\int_{p}^{\infty} \operatorname{Tr}\left(-\alpha d \delta e^{-t \Delta^{q+1}}+\alpha \delta d e^{-t \Delta^{q}}+\alpha \delta d e^{-t \Delta^{q-1}}-\alpha d \delta e^{-t \Delta^{q}}\right) d t \\
& +O\left(p^{N+1-m / 2}\right) .
\end{aligned}
$$

Note $(\ln p) \delta \zeta^{q}(0)=0$ if $M$ is odd dimensional.

The variational formula (2.5) looks substantially more complicated than the expression (1.13) for $\delta \zeta(0)$. In particular, the integral in (2.5) is a nonlocal expression, reflecting the nonlocal character of $\zeta^{\prime}(0)$. Nevertheless, in some instances we can explicitly solve the conformal Euler-Lagrange equation for $\zeta^{\prime}(0)$, which we were unable to do for $\zeta(0)$. The added control here comes from recognizing $(2.5)$ to be a precise version of a formal expression for $\delta \zeta^{\prime}(0)$. For

$$
\begin{aligned}
\int_{0}^{\infty} \operatorname{Tr}\left(-\alpha d \delta e^{-t \Delta^{q+1}}+\alpha \delta d e^{-t \Delta^{q}}+\alpha \delta d e^{-t \Delta^{q-1}}-\alpha d \delta e^{-t \Delta^{q}}\right) d t \\
\quad=\int_{0}^{\infty} \frac{1}{t} \frac{d}{d u} \operatorname{Tr}\left(e^{-t \Delta_{u}^{q}}-P_{u}\right) d t \quad \text { at } u=0 \\
=\int_{0}^{\infty} \frac{1}{t} \frac{d}{d u} \sum_{n} e^{-\lambda_{n}^{u} t} d t \quad \text { at } u=0
\end{aligned}
$$

where $\Delta_{u}^{q}$ has nonzero spectrum $\left\{\lambda_{n}^{u}\right\}$. The last integral is formally

$$
-\sum_{n} \int_{0}^{\infty} \dot{\lambda}_{n}^{u} e^{-\lambda_{n}^{u} t} d t=-\sum_{n} \frac{\dot{\lambda}_{n}}{\lambda_{n}} \quad \text { at } u=0
$$


with $\dot{\lambda}_{n}=d \lambda_{n}^{u} /\left.d u\right|_{u=0}$ and $\lambda_{n}=\lambda_{n}^{0}$. This sum is

$$
\left.\frac{d}{d u}\right|_{u=0}-\log \prod_{n} \lambda_{n}^{u}=\delta \varsigma^{\prime}(0)
$$

since $\zeta^{\prime}(0)$ is formally "minus the log of the determinant of the Laplacian" [RS, p. 151]. Thus Theorem 2.5 should be regarded as a regularization, via the added parameter $p$, of the formal expression $\delta\left(\varsigma^{q}\right)^{\prime}(0)=\int_{0}^{\infty} \operatorname{Tr} I(\alpha, q) d t$. In addition, $\int_{0}^{\infty} e^{-t \Delta} d t=\Delta^{-1}$ by the spectral theorem, at least for $\Delta$ invertible. Thus formally

$$
\delta \zeta^{\prime}(0)=-\operatorname{Tr}\left(\delta \Delta \cdot \Delta^{-1}\right) .
$$

For a finite dimensional matrix $\Delta$, the identity $-\log \operatorname{det} \Delta=-\operatorname{Tr} \log \Delta$ implies (2.6), so Theorem 2.5 should be thought of as the correct infinite dimensional analogue of $(2.6)$ for finite dimensional matrices.

We note in passing that the corresponding formal calculation for $\delta \zeta(0)$ is

$$
\begin{aligned}
\delta_{\zeta}(0) & =\frac{1}{\Gamma(s)} \int_{0}^{\infty} t^{s-1} \frac{d}{d u} \operatorname{Tr}\left(e^{-t \Delta_{u}}-P_{u}\right) d t \quad \text { at } s=0 \\
& =\frac{1}{\Gamma(0)} \cdot \delta \varsigma^{\prime}(0)=0 .
\end{aligned}
$$

In analogy with the last calculation, this may be interpreted as "correct" up to the local factors in Theorem 1.13.

Since the nonlocal term in (2.5) is unavoidable, it is useful to investigate when we can eliminate the local terms. This is always possible when $M$ is two dimensional.

THEOREM 2.7. Let $M$ be an oriented, compact, two dimensional manifold with metric $g$. For $q=0,1,2,\left(\varsigma^{q}\right)^{\prime}(0)$ is critical at $g$ for area preserving variations if and only if $g$ has constant curvature.

Thus by the uniformization theorem there is a unique critical point for $\left(\zeta^{q}\right)^{\prime}(0)$ within each conformal class of metrics. One easily checks that the theorem extends to bundles $E$ associated to representations of $\pi_{1}(M)$. In particular, when $E$ is acyclic $\sum(-1)^{q}\left(\varsigma^{q}\right)^{\prime}(0)$ is independent of the metric, but $\left(\zeta^{q}\right)^{\prime}(0)$ is never an invariant even within a conformal class.

ProOF. Let $\zeta=\varsigma^{0}$. For area preserving variations, $\delta \operatorname{res} \Gamma(1) \zeta(1)=\delta \int_{M} \operatorname{tr} C_{0}^{0}=$ $\delta$ area $(M)=0$. By $(1.17 \mathrm{~b}), \delta \operatorname{res} \Gamma(0) \zeta(0)=0$. We may now let $p$ go to zero in (2.5) to obtain $\delta \zeta^{\prime}(0)=\int_{0}^{\infty} \operatorname{Tr} I(\alpha, 0) d t$. Therefore, the actual variation agrees with the formal variation. As in $\S 1$, the Euler-Lagrange equation for $\zeta^{\prime}(0)$ is obtained by taking area preserving variations which approach a delta function $\delta_{x}, x \in M$, times the original metric. One easily checks that $\alpha$ approaches $\delta_{x}$. Id on 0 -forms and that $\operatorname{Tr} I(\alpha, 0)$ approaches $\operatorname{tr}_{x}\left(\Delta^{0} e^{-t \Delta^{0}}\right)$, the pointwise trace of the kernel of $\Delta^{0} e^{-t \Delta^{0}}$ at $x$. Thus the Euler-Lagrange equation is

$$
\int_{0}^{\infty} \operatorname{tr}_{x}\left(\Delta^{0} e^{-t \Delta^{0}}\right) d t \equiv C
$$

where $C$ is a constant arising from the area constraint.

We now simplify (2.8). 
LEMMA 2.9. Let $\Delta$ be the Laplacian on $q$-forms with pointwise zeta functions $\varsigma(s, x)$ on a manifold $M$. Then $\int_{0}^{\infty} \operatorname{tr}_{x}\left(\Delta e^{-t \Delta}\right) d t=\varsigma(0, x)$.

PROOF. Note that $\operatorname{tr}_{x}\left(\Delta e^{-t \Delta}\right)=-\partial_{t} \operatorname{tr}_{x}\left(e^{-t \Delta}\right)$. For $\operatorname{Re}(s) \gg 0$, integration by parts gives

$$
\begin{aligned}
\int_{0}^{\infty} t^{s} \operatorname{tr}_{x}\left(\Delta e^{-t \Delta}\right) d t & =s \int_{0}^{\infty} t^{s-1} \operatorname{tr}_{x}\left(e^{-t \Delta}-P\right) d t \\
& =s \int_{0}^{\infty} t^{s-1}\left(\operatorname{tr}_{x}\left(e^{-t \Delta}\right)-\beta_{q}(x)\right) d t
\end{aligned}
$$

There are no boundary contributions since $\operatorname{tr}_{x}\left(e^{-t \Delta}\right)-\beta_{q}(x)$ is exponentially decreasing as $t \rightarrow \infty$ and is $O\left(t^{-\operatorname{dim} M / 2}\right)$ as $t \rightarrow 0$. We must show that at $s=0$

$$
s \int_{0}^{\infty} t^{s-1}\left(\operatorname{tr}_{x}\left(e^{-t \Delta}\right)-\beta_{q}(x)\right) d t=\frac{1}{\Gamma(s)} \int_{0}^{\infty} t^{s-1}\left(\operatorname{tr}_{x}\left(e^{-t \Delta}\right)-\beta_{q}(x)\right) d t
$$

since the right side is $\zeta(0, x)$. Plugging in the kernel asymptotics as usual gives $\operatorname{tr}_{x} C_{m / 2}^{q}(x, x)-\beta_{q}(x)$ if $m=\operatorname{dim} M$ is even, or $-\beta_{q}(x)$ if $m$ is odd, on both sides.

Thus the Euler-Lagrange equation for $\left(\zeta^{0}\right)^{\prime}(0)$ is $\zeta(0, x) \equiv C$. Since $\beta_{0}(x)=$ $\operatorname{area}(M)^{-1}$ and $\operatorname{tr} C_{1}^{0}(x)=\tau(x) / 12 \pi$, where $\tau$ is the scalar curvature, a metric is critical for $\left(\varsigma^{0}\right)^{\prime}(0)$ if and only if it is of constant scalar curvature. The result for $\varsigma^{1}$ follows by repeating this argument on 1-forms, while the result for $\varsigma^{2}=\zeta^{0}$ is immediate.

Even the case of metrics on $S^{1}$ is not entirely trivial. All metrics of fixed length on $S^{1}$ are isometric via reparametrization by arc length, so $\left(\zeta^{0}\right)^{\prime}(0)=\left(\varsigma^{1}\right)^{\prime}(0)$ is a length invariant. It is well known that the stationary phase approximation to the path integral for the heat kernel on $\mathbf{R}$ gives the exact formula if one defines $(\operatorname{det} \Delta)^{1 / 2}$ to be $2 \pi$ on $S^{1}$. This definition coincides with the zeta function regularization $\operatorname{det} \Delta=\exp \left(-\zeta^{\prime}(0)\right)$, as we shall now compute. The following lemma simplifies a calculation due to Lerch (1894) in a more general context (cf. [C, §8]).

Lemma 2.10. For the standard metric on $S^{1}$ of length $2 \pi,\left(\varsigma^{0}\right)^{\prime}(0)=-\ln 4 \pi^{2}$.

PROOF. In this metric $\Delta^{0}=-d^{2} / d \theta^{2}$ has the orthonormal basis $\left\{\pi^{-1 / 2} \cos n \theta\right.$, $\left.\pi^{-1 / 2} \sin n \theta\right\}$ with eigenvalues $\{0,1,1,4,4, \ldots\}$. Thus $\zeta^{0}(s)=2 \sum_{n=1}^{\infty} 1 /\left(n^{2}\right)^{s}=$ $2 \zeta_{R}(2 s)$, where $\zeta_{R}$ is the Riemann zeta function. Therefore $\left(\varsigma^{0}\right)^{\prime}(0)=4 \zeta_{R}^{\prime}(0)$.

We will use the following elementary property of the Riemann zeta function $[\mathbf{R}$, $\S 38]$. For $\operatorname{Re}(s)>-1$,

$$
\zeta_{R}(s)=\frac{1}{s-1}+\frac{1}{2}+\frac{1}{12} s-\frac{1}{2} s(s+1) \int_{1}^{\infty} B_{2}(x-[x]) x^{-s-2} d x
$$

where $B_{2}(x)=x^{2}-x+1 / 6$ and $[x]$ is the greatest integer in $x$. By Taylor's formula, $\int_{1}^{\infty} B_{2}(x-[x]) x^{-s-2} d x=\int_{1}^{\infty} B_{2}(x-[x]) x^{-2} d x+O(s)$, so

$$
\zeta_{R}^{\prime}(0)=-1+\frac{1}{12}-\frac{1}{2} \int_{1}^{\infty} B_{2}(x-[x]) x^{-2} d x .
$$


We compute

$$
\begin{aligned}
\int_{1}^{\infty} B_{2}(x-[x]) x^{-2} d x= & \sum_{n=1}^{\infty}\left(\int_{n}^{n+1}(x-n)^{2} x^{-2}-(x-n) x^{-2} d x\right)+\frac{1}{6} \\
= & \sum_{n=1}^{\infty}\left(n+1-2 n \ln (n+1)-\frac{n^{2}}{n+1}+2 n \ln n\right) \\
& +\sum_{n=1}^{\infty}\left(-\ln (n+1)-\frac{n}{n+1}+\ln n+1\right)+\frac{1}{6} \\
= & \sum_{n=1}^{\infty} A_{n}+\sum_{n=1}^{\infty} B_{n}+\frac{1}{6}
\end{aligned}
$$

Now

$$
\sum_{n=1}^{N} B_{n}=-\ln (N+1)+\sum_{n=1}^{N} \frac{1}{n+1}=-\ln (N+1)+\sum_{n=1}^{N+1} \frac{1}{n}-1 .
$$

By the definition of Euler's constant $\gamma, \sum_{n=1}^{\infty} B_{n}=\gamma-1$. Also,

$$
\begin{aligned}
\sum_{n=1}^{N} A_{n}= & -2 N \ln (N+1)+2 N+\sum_{n=1}^{N}\left(-\frac{1}{n+1}+2 \ln n\right) \\
= & -2 N \ln N-2 N \ln \left(\frac{N+1}{N}\right)+2 N+\sum_{n=1}^{N}-\frac{1}{n+1}+2 \ln N ! \\
= & -2 N \ln N-2 \ln \left(1+\frac{1}{N}\right)^{N}+2 N+\left(\sum_{n=1}^{N}\left(-\frac{1}{n+1}\right)+\ln (N+1)\right) \\
& +2 \ln N !-\ln N-\ln \left(\frac{N+1}{N}\right) .
\end{aligned}
$$

In the last expression, as $N \rightarrow \infty$ the second term approaches -2 , the fourth approaches $-\gamma+1$, and the last approaches zero. Moreover, by Stirling's formula $\ln N !-N \ln N-\frac{1}{2} \ln N+N \rightarrow \ln \sqrt{2 \pi}$, so $\sum_{n=1}^{\infty} A_{n}=-2+2 \ln \sqrt{2 \pi}-\gamma+1$. Thus

$$
\int_{1}^{\infty} B_{2}(x-[x]) x^{-2} d x=-\frac{11}{6}+2 \ln \sqrt{2 \pi}
$$

and from $(2.11)$ we get $\zeta_{R}^{\prime}(0)=-\ln \sqrt{2 \pi}$.

The crucial factor of $2 \pi$ in Stirling's formula actually comes from Wallis' formula (1656)

$$
\frac{\pi}{2}=\frac{2 \cdot 2 \cdot 4 \cdot 4 \cdot 6 \cdot 6 \cdots}{1 \cdot 3 \cdot 3 \cdot 5 \cdot 5 \cdot 7 \cdots}
$$

$[\mathbf{R}]$. Following $[\mathbf{C}, \S 8]$, we will sketch a proof of Wallis' formula using the equality of analytic and Reidemeister torsion. The square of the right side of (2.12) is formally $\operatorname{det} \Delta / \operatorname{det}(\Delta+1 / 4)$ for $\Delta$ on functions on $S^{1}$. Since the right side of (2.12) converges, one easily sees that its square must equal $\exp \left(-\zeta_{\Delta}^{\prime}(0)\right) / \exp \left(-2 \zeta_{A}^{\prime}(0)\right)$, where $A=|* d|+1 / 4$ on functions on $S^{1}$. Now we know $\zeta_{\Delta}^{\prime}(0)=-\ln 4 \pi^{2}$. Using $[\mathrm{C}, \S 8]$, one checks that $\zeta_{A}(s)=\frac{1}{2} \tilde{\zeta}(s / 2)-2^{s}$, where $\tilde{\zeta}$ is the zeta function of the flat $S O(2)$ bundle associated to the representation of $\pi_{1}\left(S^{1}\right)=\mathrm{Z}$ given by $1 \rightarrow$ 
(rotation by $\pi$ ). Computing $\tilde{\zeta}^{\prime}(0)$ by the Reidemeister torsion of this bundle, we get $\zeta_{A}^{\prime}(0)=-\ln 4$. Thus the square of the right side of $(2.12)$ is $\pi^{2} / 4$.

Other, more serious, instances of the interplay between formal and rigorous expressions come from considering the eigenfunctions of the Laplacian. In fact, we can characterize flat manifolds by an eigenfunction condition and flatness at a point.

THEOREM 2.13. (a) Let $E$ be a bundle with inner product and compatible connection over $M^{n}$. Let $\left\{\phi_{i}\right\}$ be an orthonormal basis of $E$-valued $q$-forms consisting of eigenforms of the Laplacian. Then $\sum_{i} \phi_{i}(x)^{2}$ diverges for all $x \in M$. However, if $\operatorname{dim} M$ is odd, $\sum_{i}\left(\phi_{i}(x)^{2}-\phi_{i}(y)\right)^{2}=0$ whenever the sum is absolutely convergent.

(b) Let $E$ be the trivial $\mathbf{R}$-bundle.

(i) Let $\operatorname{dim} M=2$ and $q=0$. If $\sum_{i}\left(\phi_{i}(x)^{2}-\phi_{i}(y)^{2}\right)=0 \forall x, y \in M$, then $M$ has constant curvature.

(ii) Let $\operatorname{dim} M>2$. For any $q$, if $\sum_{i}\left(\phi_{i}(x)^{2}-\phi_{i}(y)^{2}\right)$ exists for points $x, y \in M$, then $\tau(x)=\tau(y)$.

(iii) Let $\operatorname{dim} M \geq 4$. $\sum_{i}\left(\phi_{i}(x)^{2}-\phi_{i}(y)^{2}\right)=0 \forall x, y \in M$ for $q=0,1,2$ and $M$ is flat at some point $x$ if and only if $M$ is flat. If $\operatorname{dim}>4$, we need only assume the sums exist and $M$ is flat at a point.

(iv) Let $\operatorname{dim} M=3 . \sum_{i}\left(\phi_{i}(x)^{2}-\phi_{i}(y)^{2}\right) \lambda_{i}^{1 / 2}=0 \forall x, y \in M$ for $q=0,1$ and $M$ is flat at some point $x$ if and only if $M$ is flat.

PROOF. (a) If $\sum \phi_{i}(x)^{2}$ exists, then so does $\sum_{\lambda_{i} \neq 0} \phi_{i}(x)^{2} \lambda_{i}^{-s}$ for $\operatorname{Re}(s)>0$. However, for $\operatorname{Re}(s) \gg 0$ this sum is $\zeta(s, x)$, which has a simple pole with residue

$$
* \operatorname{tr} C_{0}^{q}(x, x)=\left(\begin{array}{l}
n \\
q
\end{array}\right) \quad \text { at } s=\frac{n}{2}
$$

(see [G2]). Similarly, if $\sum_{\lambda_{i} \neq 0}\left(\phi_{i}(x)^{2}-\phi_{i}(y)^{2}\right)$ exists, it must equal $\varsigma(0, x)-\varsigma(0, y)$. In odd dimensions, $\zeta(0, x)-\varsigma(0, y)=-\left(\sum_{\lambda_{i}=0}\left(\phi_{i}(x)^{2}-\phi_{i}(y)^{2}\right)\right)$.

(b) If $\operatorname{dim} M=2, \varsigma^{0}(0, x)=\frac{1}{6} \tau(x)-\beta_{0}(x)=\frac{1}{6} \tau(x)-\operatorname{vol}(M)^{-1}$. This proves (i). For (ii), we note that the residue of $\varsigma(s, x)$ at $s=n / 2-1$ is a nonzero multiple of $\tau(x)$. If the sum exists, the residue of $\zeta(s, x)-\varsigma(s, y)$ must vanish.

For (iii), first let $\operatorname{dim} M=4$. Combining equations (1.6) and (1.16a), we see that $\sum_{i}\left(\phi_{i}(x)^{2}-\phi_{i}(y)^{2}\right)=0$ for $q=0,1,2$ implies $R^{2}(x)=R^{2}(y)$. (Note that (1.16a) holds pointwise for $\zeta(0, x)$, and summing over all $i$ removes the local Betti number contribution.) If $R_{i j k l}(x)=0$, then $R^{2} \equiv 0$ on $M$. Conversely, if $M$ is flat the Bieberbach Theorem implies $M$ is the quotient of the torus $T^{4}$ by a discrete subgroup. The $T^{4}$-invariance of the metric implies that the Laplacians and hence their eigenspaces are $T^{4}$-invariant. Hence the sum is zero on each eigenspace.

If $\operatorname{dim} M>4$, the right side of (1.16a) (without the integral sign) represents the residue of $\zeta(s, x)$ at $s=n / 2-2$. (The formula for the residue for two forms is the same as $\operatorname{tr} C_{2}^{2}(x, x)$ on a four manifold, which we used above.)

The sum in (iv) is the formal expression for $\zeta(n / 2-1, x)$ if $n=3$. Since $\zeta^{2}=\varsigma^{1}$ in dimension 3 , we have only two equations. Fortunately, in this dimension the Ricci tensor determines the full curvature tensor, so the result follows as above.

We can prove one more result in dimension two by considering the EulerLagrange equation for the $n$th eigenvalue of $\Delta^{0}$. Here we are viewing the $n$th eigenvalue as a suitably defined function on the space of all metrics (see [B2]). 
COROLLARY 2.14. A metric on a compact, oriented surface which is critical within its conformal class for all eigenvalues of $\Delta^{0}$ has constant curvature.

PROOF. In dimension two, a metric is critical for the $n$th eigenvalue if and only if $\sum_{i} \psi_{i}(x)^{2}$ is constant in $x \in M$, where $\left\{\psi_{i}\right\}$ is an orthonormal basis of the $n$th eigenspace [B2]. Thus $\sum_{i}\left(\phi_{i}(x)^{2}-\phi_{i}(y)^{2}\right)=0$, so part (b)(i) of the Theorem applies.

In higher dimensions the situation becomes more complicated. A metric must satisfy an increasing number of conditions for its formal variational formula to be valid. Moreover, in dimensions bigger than two the Euler-Lagrange equation involves $d \delta$ and $\delta d$-i.e. pieces of the Laplacians-so Lemma 2.9 cannot be applied. We can give some examples of when the formal variational expression is valid, and for these metrics we can write the Euler-Lagrange equation in an "explicit" nonlocal form. In the next result, a bundle associated to an orthogonal representation of $\pi_{1}(M)$ may be present, and variations are assumed to be volume preserving.

PROPOSITION 2.15. For the following metrics, the formal variation formula $\delta\left(\varsigma^{q}\right)^{\prime}(0)=\int_{0}^{\infty} \operatorname{Tr} I(\alpha, q) d t$ is valid.

(a) A constant scalar curvature metric on a manifold of dimension $n=3,4$ or 5 .

(b) The metric on a compact locally symmetric space $\Gamma \backslash G / K$ induced from the $G$-invariant metric on $G / K$.

For any metric whose formal variational formula is valid, the Euler-Lagrange equation for $\delta\left(\varsigma^{q}\right)^{\prime}(0)$ is

$$
\begin{aligned}
& -(m / 2-q+1) \operatorname{tr} d \delta g^{q+1}(x, x)+(m / 2-q) \operatorname{tr}(\delta d-d \delta) g^{q}(x, x) \\
& +(m / 2-q-1) \operatorname{tr} \delta d g^{q-1}(x, x) \equiv \text { constant } .
\end{aligned}
$$

Here $g^{k}$ is the kernel of the Green's operator $G^{k}$ on $k$-forms $\left(G^{k}\right.$ is $\Delta^{-1}$ on $d C^{\infty} \Lambda^{k-1} \oplus \delta C^{\infty} \Lambda^{k+1}$ and $G^{k}=0$ on $\left.H^{k}\right)$.

PROOF. (a) In three dimensions we have for volume preserving variations

$$
\delta\left(\zeta^{q}\right)^{\prime}(0)=p^{-1 / 2} \delta\left(\int_{M} A \cdot \tau\right)+O\left(p^{1 / 2}\right)+\int_{p}^{\infty} \operatorname{Tr} I(\alpha, q) d t .
$$

for some constant $A$. It is well known (see $[\mathbf{P 1}, \S 12]$ ) that $\delta \int_{M} \tau=0$ for all variations precisely when the metric is Einstein. Since we are only working with conformal variations, we can take the trace of the Einstein condition $\rho_{i j}=C \cdot g_{i j}$ to obtain the condition of constant scalar curvature as the Euler-Lagrange equation for conformal variations of $\int_{M} \tau$. Therefore, for these metrics $\left.\delta\left(\varsigma^{q}\right)^{\prime}(0)=\int_{0}^{\infty} \operatorname{Tr} I_{1}^{\prime} \alpha, q\right) d t$.

In dimension four, we have

$$
\begin{aligned}
\delta\left(\zeta^{q}\right)^{\prime}(0)= & p^{-1} \delta\left(\int_{M} B \cdot \tau\right)+\ln p \cdot \delta\left(\int_{M} C R^{2}+D p^{2}+E \tau^{2}\right) \\
& +\int_{p}^{\infty} \operatorname{Tr} I(\alpha, q) d t
\end{aligned}
$$

By Lemma 1.17 and the argument above, constant scalar curvature metrics are critical for conformal variations of $\int R^{2}, \int p^{2}, \int \tau^{2}$. A similar argument works in five dimensions. 
(b) By Theorem 1.18, the local terms in Theorem 2.5 vanish for a locally symmetric space.

The statement involving the Green's functions follows from Theorem 2.5 together with the identity $G^{k}=\int_{0}^{\infty} e^{-t \Delta^{k}}-P_{H^{k}} d t$, which can be seen by writing out both sides in their eigenfunction expansions.

REMARKS. (1) Of course $\operatorname{tr} g^{k}(x, x)$ does not exist. Implicit in Proposition 2.15 is the fact that for metrics whose formal variational formula is valid, (2.16) exists.

(2) One topic largely left out of this paper is critical point theory for arbitrary variations. Conformal variations lead to manipulating $\operatorname{tr} C_{k}^{q}(x, x)$, while for arbitrary variations one must consider $\operatorname{tr}\left(\alpha_{i j}\right)_{x} C_{k}^{q}(x, y)$ where the linear transformation $\left(\alpha_{i j}\right)$ is one in the $(i, j)$ slot and zero elsewhere. Unfortunately, although $C_{k}^{q}(x, y)$ has been computed for low values of $q$ and $k[\mathbf{G} \mathbf{3}]$, the resulting system of EulerLagrange equations is quite complicated.

\section{REFERENCES}

[ABP] M. F. Atiyah, R. Bott, and V. K. Patodi, On the heat equation and the index theorem, Invent. Math. 19 (1973), 279-330.

[B1] D. Bleecker, Critical Riemannian manifolds, J. Differential Geom. 14 (1979), 599-608.

[B2] _ Determination of a Riemannian metric from the first variation of its spectrum, Amer. J. Math. (to appear).

[BE] M. Berger and D. Ebin, Some decompositions of the space of symmetric tensors on a Riemannian manifold, J. Differential Geom. 3 (1969), 379-392.

[C] J. Cheeger, Analytic torsion and the heat equation, Ann. of Math. (2) 109 (1979), 259-322.

[CGW] R. Cahn, P. B. Gilkey, and J. A. Wolf, Heat equation, proportionality principle, and volume of fundamental domains, Differential Geometry and Relativity (D. Cohen and M. Flato, eds.), Reidel, Holland, 1976, pp. 43-54.

[G1] P. B. Gilkey, The spectral geometry of a Riemannian manifold, J. Differential Geom. 10 (1975), 601-618.

[G2] _ The spectral geometry of symmetric spaces, Trans. Amer. Math. Soc. 225 (1977), 341-353.

[G3] _ Recursion relations and the asymptotic behavior of the eigenvalues of the Laplacian, Compositio Math. 38 (1979), 201-240.

[MS] H. P. McKean and I. M. Singer, Curvature and the eigenvalues of the Laplacian, J. Differential Geom. 1 (1967), 43-69.

[P1] R. Palais, Applications of the symmetric critically principle to mathematical physics and differential geometry, Sonderforschungsbereich 40, Universität Bonn.

[P] V. K. Patodi, Curvature and the eigenforms of the Laplace operator, J. Differential Geom. 5 (1971), 233-249.

[R] H. Rademacher, Topics in analytic number theory, Grundlehren Math. Wiss., Band 169, Springer-Verlag, Berlin, and New York, 1973.

[de R] G. de Rham, Variétés différentiables, Hermann, Paris, 1960.

[RS] D. B. Ray and I. M. Singer, $R$-torsion and the Laplacian on Riemannian manifolds, Adv. in Math. 7 (1971), 145-210.

[T] C. L. Terng, unpublished.

Department of Mathematics, Brandeis University, Waltham, MassachuSETTS 02254

Current address: Department of Mathematics, Boston University, Boston, Massachusetts 02215 